\title{
Quand les bargers se font monnayeurs : une " aristocratie » chez les pêcheurs de l'estuaire de la Loire au XVIIIe siècle
}

\section{Vincent Bugeaud}

\section{(apenEdition Journals}

\section{Édition électronique}

URL : http://journals.openedition.org/abpo/1041

DOI : 10.4000/abpo.1041

ISBN : 978-2-7535-1500-0

ISSN : 2108-6443

Éditeur

Presses universitaires de Rennes

Édition imprimée

Date de publication : 20 décembre 2005

Pagination : 43-84

ISBN : 978-2-7535-0243-7

ISSN : 0399-0826

\section{Référence électronique}

Vincent Bugeaud, "Quand les bargers se font monnayeurs : une « aristocratie » chez les pêcheurs de l'estuaire de la Loire au XVIIIIe siècle ", Annales de Bretagne et des Pays de l'Ouest [En ligne], 112-4 | 2005, mis en ligne le 20 décembre 2007, consulté le 01 mai 2019. URL : http:// journals.openedition.org/abpo/1041; DOI : 10.4000/abpo.1041 


\title{
Quand les bargers se font monnayeurs : une " aristocratie " chez les pêcheurs de l'estuaire de la Loire au XVIII ${ }^{\mathrm{e}}$ siècle
}

\author{
Vincent BUGEAUD \\ Doctorant, CRHIA \\ Université de Nantes
}

Ce 26 avril 1732, Silvestre Chauvelon, maître pêcheur de l'île de Trentemoult, paroisse de Rezé, vient porter plainte auprès de ces messieurs les juges de l'Amirauté de Nantes. Selon lui, il aurait été en effet insulté « le jour d'hier environ les neuf à dix heures du soir " par deux autres pêcheurs, Pierre Bertrand et son fils, aussi de Trentemoult, alors qu'il était " suivant son droit à pescher dans sa barge dans la rivière de Loire". Ils lui auraient jeté plusieurs pierres dont l'une " l'ayant atteint, l'a dangereusement blessé au visage un peu au-dessous de l'œil dont il répandit beaucoup de sang ", puis ils lui auraient jeté " une bouée de filet et plusieurs autres morceaux de bois dont l'un l'atteignit au travers des reins et le renversa dans sa barge ".

Pourquoi cette violence? Pour Silvestre Chauvelon, la raison de la haine de ces pêcheurs à son égard est toute trouvée : pour lui en effet, c'est " sous prétexte qu'il est maître monnoyeur et par conséquent exempt des soudes et fouages ${ }^{1}$ en conformité des lettres patentes de Sa Majesté [que] les autres habitants de l'isle aussi pêcheurs et qui ne sont point monnoyeurs lui portent une jalousie et une haine mortelle, de telle manière qu'il ne peut pour ainsi dire aller à la pesche sans être insulté ${ }^{2}$ ".

Si on peut évidemment douter que les faits, tels qu'ils sont rapportés par Silvestre Chauvelon, soient rigoureusement exacts ${ }^{3}$, l'intérêt de ce témoignage judiciaire est de mettre en lumière l'existence, au sein de la

1. L'expression " soudes et fouages " ne paraît plus au XVIII ${ }^{\mathrm{e}}$ siècle que désigner les seuls fouages dans une formulation purement rituelle. La définition originelle des soudes renvoie peut-être à une imposition liée à l'effort de guerre.

2. Arch. dép. de Loire-Atlantique, B 4942, Plainte de Silvestre Chauvelon, 26 avril 1732.

3. Arch. dép. de Loire-Atlantique, B 4942, Information du 28 avril 1732. Il semble que Silvestre Chauvelon se soit montré quelque peu provocant envers Pierre Bertrand en lui volant "son coup de pêche". 
société des pêcheurs de l'estuaire de la Loire, d'une population s'adonnant à une biactivité dont la conjugaison apparaît a priori pour la moins originale et la singularisant nettement auprès des autres pêcheurs. Car Silvestre Chauvelon n'est pas, loin s'en faut, un cas isolé. D'après un état du personnel de l'atelier monétaire de Nantes de $1728^{4}, 41 \%$ des monnayeurs et ajusteurs de la Monnaie de Nantes, soit 43 personnes, sont rézéens, parmi lesquels on trouve une très large majorité de pêcheurs. Rezé concentrant avec Indre, comme on le verra, la quasi-totalité des pêcheurs monnayeurs.

L'historiographie a bien mis en évidence l'existence d'une biactivité au sein du monde des pêcheurs tant de mer que de rivière : pêcheurs-paysans du milieu rural, pêcheurs-artisans ou boutiquiers du milieu urbain ${ }^{5}$. L'existence en revanche d'une population de pêcheurs-monnayeurs soulève de nouvelles questions. Formant corporation à l'échelle du royaume, flanqués de privilèges, avant tout fiscaux et loin d'être symboliques, ces monnayeurs forment une véritable société dans la société des pêcheurs d'estuaire, avec les jalousies que l'on peut supposer, et les "malheurs " de Silvestre Chauvelon en sont un témoignage.

Pour approcher cette population, il va donc falloir d'abord s'efforcer de saisir les caractéristiques, très particulières, de son statut et de son travail au sein de l'atelier monétaire de Nantes, pour étudier ensuite la place qu'elle occupe au sein de la corporation des monnayeurs, appelée le "Serment de France ", et comment elle peut concilier l'activité de monnayage, avec ses contraintes, et l'activité pêche, qui a aussi les siennes. Enfin, il s'agira d'approcher le positionnement social singulier de ces pêcheurs-monnayeurs au sein de la société des pêcheurs d'estuaire et à l'échelle paroissiale ${ }^{6}$.

4. Arch. mun. de Nantes, HH 25, pièce 37, Liste de monnoyeurs, ajusteurs, tailleresses et veuves de la monnoye de Nantes, 26 février 1728.

5 . Une première approche du concept de pluriactivité appliqué aux sociétés littorales a été proposée par Gérard LE BOUEDEC : "La pluriactivité dans les sociétés littorales, XVII ${ }^{\mathrm{e}}$ $\mathrm{XIX}^{\mathrm{e}}$ siècles ", Annales de Bretagne et des Pays de l'Ouest, tome 109, n 1, 2002, p. 61-90. Approche approfondie ensuite par un colloque : LE BouEdEc, Gérard, Ploux, François, CERINO, Christophe, GEISTDOERFER, Aliette (dir.), Entre terre et mer : sociétés littorales et pluriactivités ( $X V^{e}-X I X^{e}$ siècles), Rennes, PUR, 2004, 391 p. Pour le monde rural littoral et ses pêcheurs-paysans, pour donner des exemples bretons, citons la thèse de Dominique RoBIN sur les pêcheurs de sardines du Sud-Bretagne : Pêcheurs bretons sous l'Ancien Régime, l'exploitation de la sardine sur la côte Atlantique, Rennes, PUR, 2000, 387 p., et celle d'Olivier Levasseur sur le Trégor : LevasseuR, Olivier, Les Usages de la mer dans le Trégor au XVIII siècle, thèse de doctorat, Université de Rennes 2, 2000, 1631 p. Concernant les gens de Loire, ceux en amont de Nantes, nous disposons d'un mémoire de maîtrise : Molx, Bruno, Gens de Loire au XVIII siècle : Hérédité professionnelle et niveau de vie, mémoire de maîtrise, Yves Durand (dir.), Université de Nantes, 1983, 219 p. Au travers d'un exemple rural (Anetz) et urbain (Ancenis), l'auteur a mis en évidence le profil du pêcheur-laboureur du monde rural et du pêcheur-marinier ou artisan du monde urbain.

6. Aucune étude n'a été consacrée aux pêcheurs-monnayeurs. La biactivité de cette population a seulement été identifiée par une poignée de travaux. Citons, pour Rezé : KERVAREc, Michel, Terroir et Moyen Âge au pays nantais. Rezé, Les Sorinières, Vertou, Nantes, Editions du Petit Véhicule, 1999; pour Indre : LoDE, Corinne, Etude d'une communauté 


\section{Le Serment de France}

Avant de cerner la place que les pêcheurs occupent au sein de l'atelier monétaire, il est nécessaire, au préalable, de décrire les caractéristiques du personnel et de son travail, la spécificité de ce dernier rendant nécessaire l'exercice d'une activité complémentaire, pour ne pas dire principale.

\section{Le personnel de la Monnaie}

Si l'on suit les chiffres de frappes donnés par Michel Morineau, l'atelier nantais semble être au $\mathrm{XVIII}^{\mathrm{e}}$ siècle d'une importance "moyenne " en comparaison de l'ensemble des autres ateliers, modeste même face aux quelques grands ateliers du Royaume qui concentrent la plus grande partie des frappes ${ }^{8}$. Le personnel de cet atelier donc « moyen " sinon modeste

rurale au XVIII siècle : Indre, mémoire de maîtrise, Guy Saupin (dir.), Université de Nantes, 1992, ainsi qu'une thèse de doctorat en droit : LE BASTARD, Hubert, Les Pilotes lamaneurs ligériens. Essai sur le recrutement et l'encadrement du personnel du pilotage sous le régime de l'Ordonnance de Marine d'août 1681 (1681-1806), thèse de doctorat en droit maritime, Université de Nantes, 1976, 459 p. Cette dernière mettant en évidence le profil de pilotemonnayeur sur lequel nous reviendrons.

7. On ne peut que constater le quasi-vide historiographique concernant l'étude sociale du personnel de la Monnaie sous l'Ancien Régime. Deux catégories essentielles de chercheurs, à la frontière parfois floue, se sont jusqu'ici intéressées aux ateliers monétaires. Les numismates d'abord, qui se focalisent naturellement sur l'étude des frappes monétaires : sur ce point, les études sur les ateliers monétaires (sur)abondent, mais généralement en dehors de la sphère universitaire. Les historiens économistes d'autre part, qui se consacrent eux aussi à l'analyse des frappes mais pour étudier la circulation monétaire; sur ce point-là, les études sont nettement plus rares. De cette faible production universitaire, on retiendra deux thèses qui accordent une large place à l'étude du personnel, mais sans sortir ou presque des quatre murs de l'atelier : PlANCHENAULT, Adrien : La Monnaie d'Angers, origines, la Monnaie Royale (1319-1738), la juridiction de la Monnaie jusqu'en 1791, Angers, Lachèse et C ${ }^{\text {ie }}, 1896,236$ p. ; Collin, Bruno, L'Atelier monétaire royal de Montpellier et la circulation monétaire en Languedoc de Louis XIII à la Révolution, 16101793, B. Collin, 1986, 503 p., cette dernière constituant l'étude de référence. Pour les ateliers bretons, les médiévistes ont été bien plus actifs que les modernistes, on citera : KERHERVE, Jean, L'État breton aux XIV et XV siècles. Les ducs, l'argent et les hommes, Paris, Malouine, 1987, 1078 p.; CoAtıvy, Yves, La Monnaie en Bretagne de l'an Mil à 1499, thèse de doctorat, Jean Kerhervé (dir.), Université de Bretagne occidentale, 1999, 1447 p. Pour l'Ancien Régime, il n'existe aucun travail universitaire spécifiquement consacré aux ateliers de Rennes et Nantes. On notera pour Rennes, une étude motivée par des recherches généalogiques mais présentant une synthèse sur l'organisation de l'atelier rennais : AUBREE, Edouard, Une Famille de monnoyers rennais aux XVI ${ }^{e}$ XVII et XVIII siècles. Étude sur la Monnaie de Rennes, Rennes, Simon, 1903, 328 p. Pour Nantes, une approche a été proposée par Paul Manceron : MANCERON, Paul, " L'Atelier monétaire de Nantes, notes contributives à son histoire ", Bulletin de la société archéologique et historique de Nantes et de Loire-Inférieure, tome 91, 1952, p. 1-26. Citons enfin l'existence d'une liste, partielle, de monnayeurs nantais : GRANGes DE SuRGEREs, Marquis des, Les Artistes nantais du Moyen Âge à la Révolution, Paris, Charavay frères, 1899, 456 p.

8. Pour tenter quelques délicates comparaisons ponctuelles : en 1738, Nantes occuperait le $14^{\mathrm{e}}$ rang sur 29 ateliers, soit 1,8\% des frappes avec un bémol néanmoins, le volume des frappes d'argent nantaises, sans doute faible, n'étant pas connu. Les 10 premiers ateliers du royaume totaliseraient 73,4 \% des frappes. En 1775, sur les 17 ateliers 
peut se diviser en trois catégories : les officiers de fabrication, les officiers de juridiction et le personnel d'exécution c'est-à-dire le Serment de France. Nous n'évoquerons que très rapidement les deux premières catégories qui ne concernent pas la population de pêcheurs mais avec lesquelles elle se trouve évidemment en relation.

Les officiers de fabrication sont le directeur de la Monnaie, chargé de l'administration de la Monnaie ${ }^{9}$, l'essayeur chargé des vérifications, et le graveur chargé de la réalisation des poinçons. Le personnel de juridiction est composé, lui, d'un général provincial des Monnaies en Bretagne : à la tête d'un département comprenant les évêchés de Nantes, Vannes et Quimper, c'est l'autorité juridictionnelle suprême de l'Hôtel des Monnaies, il constitue le lien entre cette dernière et la Cour des Monnaies de Paris. Il est assisté de deux juges-gardes, juges spéciaux de la Monnaie, et comme la confiance règne, d'un contrôleur contre-garde, surveillant les deux autres. Dans les faits, les juges-gardes sont les hommes du général provincial, le contre-garde, l'homme du directeur de la Monnaie... Â cela se rajoutent comme dans toute juridiction, un avocat du Roi, un procureur du Roi, des greffiers et huissiers.

Intéressons-nous maintenant au Serment de France. Ce dernier est une vaste corporation, unique à l'échelle du royaume, qui regroupe l'ensemble du personnel d'exécution des ateliers monétaires. D'après Adrien Planchenault, " ce nom est tiré du serment solennel que les rois de la seconde race avaient, afin de réprimer les désordres des monnayeurs mérovingiens, rendu obligatoire pour les ouvriers dans leurs ateliers ${ }^{10}$ ". Il existait en fait au Moyen Âge différents Serments, les deux plus importants étant le Serment de France et celui d'Empire qui semblent avoir cohabité en Bretagne ${ }^{11}$. Ces différents Serments ont fusionné en un seul Serment de France en $1541^{12}$.

Le personnel du Serment de France se divise en deux groupes : les officiers et le personnel d'exécution proprement dit. Ce dernier groupe ras-

restant en activité après la réforme de 1772, les 5 premiers ateliers concentreraient 71,9\% des frappes, Nantes n'occupant que le $12^{\mathrm{e}}$ rang avec $1,1 \%$ des frappes constituées de 85,9 \% d'or. Pour la période 1776-1780, Nantes représente 1,39 \% des frappes, composées de 79,2 \% d'or. De cet exercice hasardeux consistant à comparer ponctuellement une activité par définition aléatoire, déduisons cependant sans grand risque que l'atelier nantais au XVIII ${ }^{\mathrm{e}}$ siècle ne fait pas partie du peloton de tête des quelques grands ateliers du royaume qui concentreraient plus des deux tiers des frappes. MoRINEAU, Michel, "Les frappes monétaires françaises de 1726 à 1793. Premières considérations ", dans DAY, John (dir.), Etudes d'histoire monétaire, Lille, Presses universitaires de Lille, 1984, p. 69-141.

9. Gérard Mellier, maire de Nantes de 1720 à 1729, a été directeur de la Monnaie en 1697. Notons également le cas de ce Lorgerie, condamné par contumace en 1709 à être " pendu et estranglé " pour une série de détournement " d'espèces et matières " au sein de la Monnaie... avec quelques complicités. Arch. dép. d'Ille-et-Vilaine, C 1864, Sentence contre les officiers de la Monnoye de Nantes, 29 mai 1709.

10. Planchenault, Adrien, op. cit., p. 78.

11. KERHERVE, Jean, op. cit., p. 200-201.

12. Collin, Bruno, op. cit., p. 38. 
semble l'ensemble des monnayeurs, ajusteurs et tailleresses. Les ajusteurs ${ }^{13}$ ont pour rôle de préparer les pièces pour la frappe : ils pèsent les flans sur la balance. S'ils sont trop lourds, ils les ajustent, s'ils sont trop légers, ils les renvoient à la fonte. Les ajusteurs existent en version féminine : ce sont les tailleresses, dont le rôle est également de réduire les pièces au poids fixé par les ordonnances. Une fois les flans préparés, c'est le monnayeur qui se charge de la frappe. $\mathrm{Au} \mathrm{XVIII}^{\mathrm{e}}$ siècle, celle-ci se fait au balancier.

Ce personnel est encadré par des officiers du Serment de France : il s'agit d'un prévôt secondé par un lieutenant, la chambre du monnayage et celle de l'ajusterie en possèdent chacune un. Le rôle de ces officiers est d'administrer l'ensemble du personnel du Serment et d'assurer le cas échéant la police. Issus du Serment, notons également l'existence d'un syndic représentant les deux chambres, probablement auprès du personnel de juridiction, ainsi que d'un vérificateur. Enfin, on relève l'existence, mais pour le début de notre période d'étude seulement, d'une confrérie de SaintLouis, à l'utilité énigmatique, dirigée par deux prévôts. En 1708, ces deux prévôts sont des pêcheurs : Yves Saulny et César Chauvelon ${ }^{14}$.

\section{Entrer et évoluer au Serment de France}

Ne rentre pas à la Monnaie qui veut. Il existe trois moyens d'admission : l'hérédité, la création par événement royal et la création en masse dictée par la nécessité ${ }^{15}$. Les deux dernières possibilités sont exceptionnelles, la première est la règle ${ }^{16}$. Pour intégrer le Serment de France, il faut être " d'estoc et de droite ligne de monnaie ". L'hérédité ne joue qu'en ligne directe, jusqu'au deuxième degré seulement. En cas d'interruption sur deux générations, le postulant au Serment a cependant la possibilité de demander une lettre d'interruption au Roi ${ }^{17}$.

13. Ou " ouvriers ", terme que l'on trouve dans le cadre chronologique de notre étude pour la fin XVII ${ }^{\mathrm{e}}$ siècle et le début du XVIII ${ }^{\mathrm{e}}$ siècle. Il s'efface par la suite au profit du terme " ajusteur ", simple évolution de vocabulaire ne se traduisant manifestement pas par un changement de fonction au sein de l'atelier.

14. Arch. dép. de Loire-Atlantique, B 5216, Audience du 26 mars 1708, f ${ }^{\circ} 47$. Sauf indication contraire, tous les monnayeurs cités en exemple appartiennent au monde des bargers de l'estuaire de la Loire.

15. Planchenault, Adrien, op. cit., p. 79.

16. Un exemple de création par évènement royal : en mars 1656, la promulgation d'un édit de Louis XIV "portant création en chacune Monnoye de ce Royaume, en faveur du Sacre de sa Majesté, d'un ouvrier et d'un monnoyeur du Serment de France... ", Arch. dép. de Loire-Atlantique, B 5276; les ducs de Bretagne procédaient de même, KERHERVE, Jean, op. cit., p. 201.

17. Ces lettres sont présentes dans les registres du greffe de l'hôtel des Monnaies. Ainsi, celle de Guillaume Saulny datant de 1708 : ce dernier y démontre sa filiation " monnayeuse " sur 132 ans! Arch. dép. de Loire-Atlantique, B 5216, Lettre d'interruption de Guillaume Saulny, 14 mai $1708, \mathrm{f}^{\circ} 45 \mathrm{r}^{\circ}$. Sources précieuses de renseignements pour la reconstitution de lointaines filiations « monnayeuses " interrompues, elles sont un complément indispensable aux registres paroissiaux et aux réceptions, surtout si ces derniers sont lacunaires. 
Les sentences des réceptions à la Monnaie de Nantes nous renseignent sur les différents critères à respecter en fonction du métier envisagé au sein du Serment ${ }^{18}$. Devenir monnayeur est réservé au fils aîné de monnayeur ou ajusteur, ajusteur au fils cadet. Pour devenir tailleresse, il faut être fille de monnayeur ou de tailleresse, ces dernières sont alors appelées " seconde filles ${ }^{19}$ ". Il est possible également pour les femmes de monnayeur ou d'ajusteur de devenir tailleresse sauf si, devenues veuves, elles se remarient avec des personnes étrangères au Serment ${ }^{20}$. Tout candidat au Serment faisant preuve de sa filiation peut donc être reçu, c'est-à-dire qu'il peut y avoir une totale inadéquation entre les effectifs du Serment et le niveau d'activité de l'atelier ${ }^{21}$.

Sur un échantillon de 101 noms ${ }^{22}$, l'âge moyen d'accession au Serment est à la vingtième année.

Les monnayeurs semblent donc pour l'essentiel accéder logiquement au Serment dès qu'ils sont en âge de pouvoir y postuler. Certains y parviennent même dès l'âge de 12 ou 13 ans : pour citer un pêcheur, c'est le cas de César Silvestre Chauvelon en $1713^{23}$, mais plus sûrement à 16 ans, ce qui est le cas par exemple de Simon Pierre Peillac, père et fils, respectivement en $1733^{24}$ et $1759^{25}$. Pour les plus âgés, le maximum retrouvé dans le sondage ne dépasse pas 33 ans.

L'accession au Serment ou " accueillement " se fait après demande écrite de l'intéressé où il doit exposer son droit de filiation. Il doit également fournir un certificat " de bonnes mœurs et catholicité ", ce dernier étant signé par deux ou trois personnes : ce sont généralement des maîtres ou officiers

18. Les sentences des réceptions à l'atelier monétaire de Nantes sont conservées aux Archives départementales de Loire-Atlantique : B 5290 à B 5369, procédures et pièces annexes (1577-1790). Seules les sentences pour la fin du XVII ${ }^{\mathrm{e}}$ siècle et le XVIII ${ }^{\mathrm{e}}$ siècle ont été conservées avec quelques lacunes mineures. On notera une erreur de classement qui a fait échouer certaines réceptions dans le fonds du siège royal des Traites.

19. Ces "secondes filles " semblent disparaître au cours du XVIII ${ }^{\mathrm{e}}$ siècle, victimes d'une évolution réglementaire, AUBREE, Edouard, op. cit., p. 44.

20. Planchenault, Adrien, op. cit., p. 80.

21. Pour citer une lettre du prévôt des ajusteurs de la Monnaie de Paris, datant de 1719 : " le nombre des monoyeurs et ouvriers n'est point fixe, toux ceux qui sont d'estoc et de ligne qui se présentent et font leurs filiations nous les recevons ", Collin, Bruno, op. cit., p. 469.

22. Un sondage dans le fonds des réceptions de l'atelier monétaire a permis de constituer un échantillon de 155 noms de monnayeurs, pêcheurs et non-pêcheurs, plus 10 noms de tailleresses, peu nombreuses en dehors de la bourgeoisie. Dans ces 155 noms, l'âge d'accession au Serment est précisé pour 101 personnes. Les bourgeois nantais postulant au Serment, " anomalie " sur laquelle nous reviendrons, n'ont pas été retenus pour ne pas fausser la moyenne, ces derniers accédant au Serment à un âge généralement plus tardif.

23. Arch. dép. de Loire-Atlantique, B 5309, Réception de César Silvestre Chauvelon, monnayeur, 9 septembre 1713 .

24. Arch. dép. de Loire-Atlantique, B 5332, Réception de Simon Pierre Peillac, monnayeur, 21 novembre 1733.

25. Arch. dép. de Loire-Atlantique, B 5352, Réception de Simon Pierre Peillac, monnayeur, 19 décembre 1759 . 
Figure 1 - Répartition de l'âge d'accession au Serment de France nantais au XVIII siècle

(échantillon de 101 monnayeurs et ajusteurs)

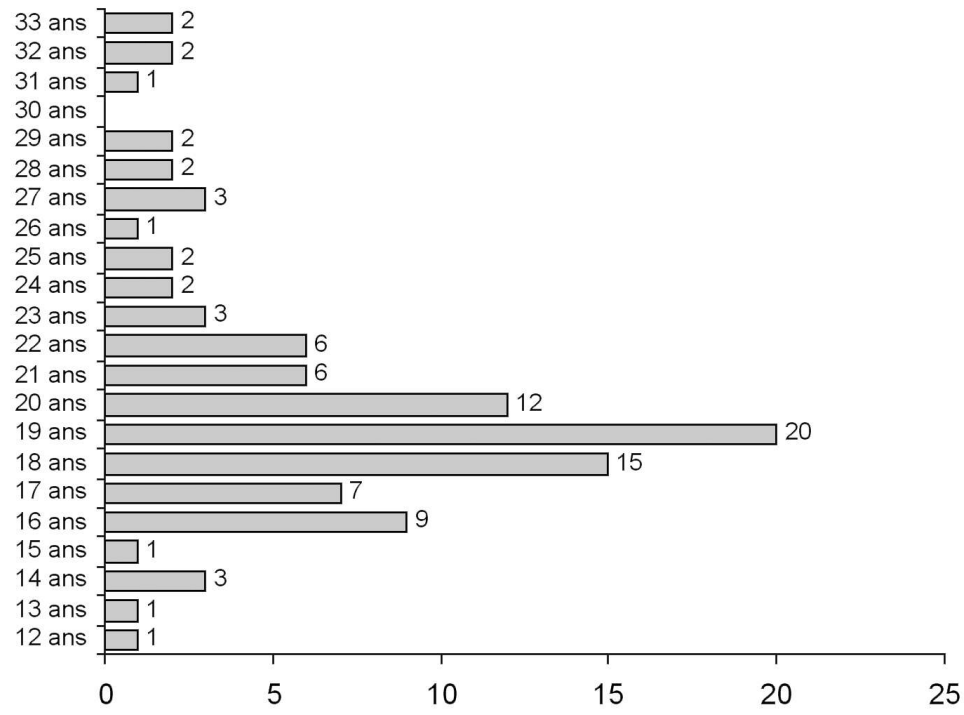

du Serment, pas forcement de la même paroisse ou profession, parfois des prêtres ou notables ${ }^{26}$. Le nouvel ouvrier apprenti peut alors être reçu en la chambre du monnayage s'il est fils aîné, dans celle de l'ajusterie, s'il est fils cadet.

En 1704, un fait montre la considération plus haute dévolue à la chambre des monnayeurs, apanage des aînés, sur celle de l'ajusterie. En effet, cette année-là, Mathurin Ertaud, qui s'était fait recevoir en la chambre de l'ajusterie en 1693 demande à passer maître non pas dans cette même chambre mais dans celle du monnayage : son frère aîné étant mort " en l'isle de la Guadeloupe ", sa réception en la chambre de l'ajusterie a été par conséquent, pour lui, une erreur. Pour appuyer sa demande, il souligne des cas similaires, mais eux vraisemblablement volontaires, montrant un respect du règlement qui n'est pas des plus scrupuleux. Il cite notamment l'exemple d'un autre pêcheur, Pierre Peillac, " entré par usurpation dans le monnoyage car Silvestre Peillac, son frère aîné, est dans le même monnoyage et reçu avant ${ }^{27}$ ". Il réussit finalement à accéder à la chambre du

26. Par exemple, pour la réception du pêcheur rézéen Jacques Flamant, c'est le « noble et discret " Pierre Geslin, " prestre chanoine de Notre-Dame de Nantes " qui apporte sa caution, Arch. dép. de Loire-Atlantique, B 5338, Réception de Jacques Flamant, ajusteur, 26 juillet 1738; Pour celle d'un autre pêcheur rezéen, Silvestre Dejoie, c'est le notaire trentemousin Fruneau, alors âgé de 72 ans, qui apporte la sienne, Arch. dép. de LoireAtlantique, B 5300, Réception de Silvestre Dejoie, 16 janvier 1703.

27. Arch. dép. de Loire-Atlantique, B 5301, Requête de Mathurin Ertaud, 5 décembre 1701. 
monnayage : Mathurin Ertaud est le seul exemple de "transfert " constaté entre les deux chambres ${ }^{28}$.

Pour accéder à la maîtrise, il faut bien entendu exécuter un chefd'œuvre. Celui-ci ne change pas au cours du XVIII ${ }^{\mathrm{e}}$ siècle. Pour les monnayeurs, cela correspond à monnayer sous le contrôle d'un parrain, 3 ou 4 marcs d'écus de 6 livres. En 1738, Simon Pierre Peillac doit ainsi monnayer " 4 marcs d'écus sous le balancier, après l'avoir démonté seul et aussi remonté seul sous l'inspection de César Silvestre Chauvelon, Maître Monnoyeur ${ }^{29}$ ". Pour les ajusteurs, le chef-d'œuvre consiste, en règle générale, à ajuster 4 marcs de flans d'écus de 6 livres. Il y a cependant quelques exceptions : en 1778, par exemple, le chef-d'œuvre de Pierre Aubin a consisté à ajuster " 2 marcs de flans bruts de Louis d'or de 48 livres ", il a pour parrain Yves Peillac père, " ancien Maître ajusteur ${ }^{30}$ ".

Concernant l'âge d'accession à la maîtrise, une moyenne, mais sur 52 noms seulement ${ }^{31}$, indique un âge d'accession moyen à la $26^{\mathrm{e}}$ année, ce qui tendrait donc à montrer que les ouvriers accèderaient à la maîtrise généralement peu de temps après avoir été reçus au Serment, ce que confirme un recoupement entre les dates d'accession au Serment et à la maîtrise. En effet, sur 96 réceptions à la maîtrise, il apparaît qu'un peu plus du tiers des ouvriers accèdent à la maîtrise dans les deux années qui suivent l'accession au Serment, ils sont un peu plus des deux tiers à y accéder dans les cinq années qui suivent. Pour certains, cela va très vite : c'est le cas de Jean Dejoie, devenant maître monnayeur qu'un peu plus de treize mois après avoir été reçu au Serment ${ }^{32}$. Pour d'autres, c'est bien plus long : Silvestre Dejoie doit patienter vingt-quatre ans avant d'être reçu maître ajusteur en 1755, il a alors 42 ans $^{33}$. Et on peut penser que quelques-uns ont très bien pu mourir de vieillesse sans avoir été reçu maître...

Le cas des vingt-quatre ans " d'attente » de Silvestre Dejoie est en fait révélateur d'une évolution réglementaire qu'il faut signaler ici. En 1755, en effet, un arrêt du Conseil d'État, qui généralise à l'ensemble de la corporation une sentence concernant l'Hôtel des Monnaies de Besançon et visant à pallier une insuffisance du recrutement sur lequel on reviendra, déclare « déchus pour

28. Arch. dép. de Loire-Atlantique, B 5301, Réception de Mathurin Ertaud, maître monnayeur, 21 mai 1704.

29. Arch. dép. de Loire-Atlantique, B 5338, Réception de Simon Pierre Peillac, maître monnayeur, 14 août 1738.

30. Arch. dép. de Loire-Atlantique, B 5361, Réception de Pierre Aubin, maître ajusteur, 4 mars 1778. Le terme " ancien maître ajusteur/monnayeur ", rencontré différentes fois, est ici une marque d'ancienneté; il ne signifie nullement que le monnayeur a quitté le Serment.

31. Les sentences des réceptions à la maîtrise mentionnent la date d'accession au Serment mais pas l'âge du reçu : il faut donc pouvoir recouper systématiquement ces dernières avec les sentences des réceptions au Serment.

32. Arch. dép. de Loire-Atlantique, B 5304, Réception de Jean Dejoie, maître ouvrier, 16 juillet 1707 .

33. Arch. dép. de Loire-Atlantique, B 5349, Réception de Silvestre Dejoie, maître monnayeur, 6 octobre 1755 . 
toujours de leurs droits et privilèges ceux qui à l'avenir ayant droit aux dites places [de monnayeurs, ajusteurs et tailleresses] ne se feront pas accueillir dans le cours de l'année dans laquelle ils auront atteint l'âge requis par les ordonnances ", de plus cet arrêt " déclare pareillement déchus pour toujours de tous droits et privilèges ceux qui ne se feront pas recevoir aux dites places dans le courant de six mois après l'année finie de leur accueillement ou apprentissage $^{34}$ ". Arrêt rectifié quelques mois plus tard par un arrêt de la Cour des Monnaies qui fixe le délai limite de l'accueillement à l'année qui commencera le jour où les ouvriers auront atteint l'âge de 25 ans accomplis, âge limite qui n'avait pas été précisé dans l'Arrêt du Conseil d'État ${ }^{35}$. Si on suit Édouard Aubrée, cette nouvelle réglementation ne semblerait pas avoir été un coup d'épée dans l'eau puisqu'un regain des réceptions paraît observable à Rennes ${ }^{36}$. Les 42 réceptions conservées pour l'année 1756 confirmeraient également ce regain, du moins à court terme, pour l'atelier nantais ${ }^{37}$.

Le cas des tailleresses semble bien différent. Elles apparaissent peu nombreuses au XVIII ${ }^{\mathrm{e}}$ siècle, tout du moins si l'on ne tient pas compte des tailleresses de la bourgeoisie nantaise. L'âge d'accession au Serment apparaît plus tardif que celui des ouvriers. Dans le cadre de notre sondage, 10 tailleresses, hors bourgeoisie, ont été identifiées : elles sont toutes reçues à plus de 40 ans sauf une. La plus âgée est reçue à 55 ans $^{38}$ et la plus jeune, Jeanne-Baptiste Peillac, qui constitue donc une exception dans la population sondée, est reçue à 26 ans $^{39}$. Pourquoi devenir tailleresse à un âge si tardif? La raison principale, si ce n'est exclusive, est le désir de ces femmes de transmettre leurs droits à leurs enfants. Le mariage avec une tailleresse potentielle permet en effet à une famille non " monnayeuse " de faire son entrée à la Monnaie par les enfants de cette dernière, à condition qu'elle se fasse recevoir. Il n'est donc pas étonnant de voir les tailleresses faire leur entrée au Serment en même temps ou presque que leur fils aîné. C'est ainsi que toutes les tailleresses recensées, si on excepte JeanneBaptiste Peillac, ont un enfant qui se fait recevoir dans les jours suivant leur réception, si ce n'est le jour même : Magdelaine Dejoie est reçue tailleresse le 15 février 1734 à 42 ans; son fils, Joseph Aubin, âgé de 19 ans, se fait recevoir dès le lendemain monnayeur, créant ainsi une nouvelle branche Aubin " monnayeuse ${ }^{40}$ "; Julienne Peillac, elle, est reçue le 3 avril

34. Arch. dép. de Loire-Atlantique, B 5221, Arrêt du Conseil d'État du 28 octobre 1755, $\mathrm{f}^{\circ} 73 \mathrm{v}^{\circ}-\mathrm{f}^{\circ} 75 \mathrm{r}^{\circ}$.

35. Arch. dép. de Loire-Atlantique, B 5221, Arrêt de la Cour des Monnaies du 17 janvier $1756, \mathrm{f}^{\circ} 75 \mathrm{v}^{\circ}-\mathrm{f}^{\circ} 76 \mathrm{v}^{\circ}$.

36. AUBREE, Edouard, op. cit., p. 51.

37. Arch. dép. de Loire-Atlantique, B 5164.

38. Arch. dép. de Loire-Atlantique, B 5304, Réception de Ollive Ertaud, tailleresse, 6 avril 1707. Ollive Ertaud appartient à une branche d'Ertaud laboureurs-monnayeurs de Rezé et est mariée à un Julien Alain, tonnelier.

39. Arch. dép. de Loire-Atlantique, B 5351, Réception de Jeanne-Baptiste Peillac, tailleresse, 5 janvier 1758. Son âge " précoce " de réception est sans doute lié à l'évolution réglementaire. 40. Arch. dép. de Loire-Atlantique, B 5333, Réception de Magdelaine Dejoie, tailleresse, 15 février 1734; Réception de Joseph Aubin, monnayeur, 16 février 1734. 
1697 et son fils se fait recevoir le jour même ${ }^{41}$. Comme le seul souci des tailleresses est la conservation et la transmission de leurs droits à leurs enfants, elles ne sont guère intéressées à accéder à la maîtrise, possibilité qu'elles ont pourtant. Les seuls cas recensés de maîtresses tailleresses ne concernent que la bourgeoisie nantaise et sont postérieurs aux arrêts de 1755 et $1756^{42}$.

Le profil dressé ici ne permet donc pas vraiment de considérer les tailleresses comme des ouvrières effectives. Elles n'ont d'ailleurs jamais été rencontrées dans des documents autres que les réceptions et rôles de personnel... Le rôle d'une tailleresse est donc avant tout de créer une nouvelle filiation de monnayeurs.

Il y a un cas qu'il faut évoquer ici puisqu'il concerne quelques pêcheurs au XVIII ${ }^{\mathrm{e}}$ siècle, c'est celui des conditions d'accession au niveau des officiers du Serment de France ${ }^{43}$. Que cela soit prévôt ou lieutenant, il s'agit d'une fonction à vie, celle-ci s'obtenant par cooptation des maîtres ${ }^{44}$. Une parenthèse doit cependant être signalée : un édit de janvier 1705 créé en effet des offices de prévôt et de lieutenant dans chaque atelier, mais avec un tel insuccès que l'ancien système électif est rétabli officiellement en $1717^{45}$.

Il semble assez naturel que cela soit le lieutenant qui succède au prévôt. C'est ainsi, par exemple, que les choses se passent en 1764 lors de l'élection du nouveau prévôt des monnayeurs, Guillaume Peillac qui était jusque là lieutenant des monnayeurs : "Les Maîtres monnoyeurs [...] assemblés [...] ont déclaré unanimement élire pour leur prévôt Guillaume

41. Arch. dép. de Loire-Atlantique, B 5294, Réception de Julienne Peillac, tailleresse, 3 avril 1697; Réception de Guillaume Dejoie, monnayeur, 3 avril 1697. Autre exemple dans le tableau en annexe avec Marie Peillac.

42. À l'exemple de Jeanne Richeux, épouse du négociant Thomas White, reçue maîtresse tailleresse en 1757. Celle-ci " exécute ", si on peut dire, son chef-d'œuvre consistant à « ajuster le nombre de 6 marcs d'écus de 6 livres pièce par elle seule et sans l'aide de personne ", Arch. dép. de Loire-Atlantique, B 5350, Réception de Jeanne Richeux, maîtresse tailleresse, 16 mai 1757 . Notons qu'en cette même année 1757, une réglementation de l'atelier de Rennes supprime la maîtrise pour les tailleresses, une tailleresse "n'étant presque point dans l'usage de travailler, et ne se faisant recevoir que pour donner droit à ses enfants ", citée par AuBreE, Edouard, op. cit., p. 59.

43. Au XVIII ${ }^{\mathrm{e}}$ siècle, cela semble concerner quatre pêcheurs rezéens, peut-être un peu plus. Deux frères Ertaud : André Ertaud, prévôt des monnayeurs (1745) et Jean Ertaud, prévôt des ajusteurs (1733); et deux Peillac : Guillaume Peillac, lieutenant (1750) puis prévôt des monnayeurs (1764); Yves Peillac, lieutenant (1733) puis prévôt des ajusteurs (1750).

44. Il faut noter néanmoins une possibilité pour ces derniers de révoquer par la suite les deux officiers, le contrôle serait donc réciproque, Collin, Bruno, op. cit., p. 176. Ce dernier cite des exemples pour Montpellier. En revanche, le sondage effectué sur le XVIII ${ }^{\mathrm{e}}$ siècle n'a pas révélé d'exemple de révocation pour l'atelier nantais.

45. Arch. dép. de Loire-Atlantique, B 5220, Édit du Roy portant suppression des Offices..., février $1717, \mathrm{f}^{\circ} 73-\mathrm{f}^{\circ} 76$. Ces officiers, non élus par les monnayeurs, imposés de l'extérieur, avaient quelque peu de mal à se faire respecter : à Montpellier, " [les] ouvriers et monnayeurs cessèrent fréquemment le travail ou arrivèrent systématiquement en retard, contestant à tout propos l'autorité et les décisions des officiers ", Coluin, Bruno, op. cit., p. 176. À Nantes, le système électif est rétabli de fait dès 1709 , Arch. dép. de LoireAtlantique, B 5218, Élection du prévôt et lieutenant de l'ouvrairie, 9 avril $1709, \mathrm{f}^{\circ} 4$. 
Peillac, actuellement leur lieutenant. " La place de lieutenant étant vacante, c'est un nantais, sans doute marchand de grains ${ }^{46}$, qui est élu : " Après avoir été à la picque, il s'est trouvé que le sieur François Jacques Couillaud Dubreil, Maître Monnoyeur, a été le plus grand nombre de suffrages pourquoy il a été élu lieutenant des dits monnoyeurs ${ }^{47}$."

Lorsque les monnayeurs sont reçus ouvriers et maîtres ou élus officiers, ils doivent bien sûr acquitter des droits. Ceux-ci, fort divers d'un atelier à l'autre, ont été unifiés à la baisse en $1754^{48}$ et relevés en $1779{ }^{49}$.

Tableau 1 - Montant total des droits acquittés par le personnel du Serment de France en 1754 et 1779

\begin{tabular}{|l|c|c|}
\hline & 1754 & 1779 \\
\hline $\begin{array}{l}\text { Accueillement des monnayeurs, } \\
\text { ajusteurs et tailleresses }\end{array}$ & 28 livres 10 sols & 32 livres 10 sols \\
\hline $\begin{array}{l}\text { Réception des monnayeurs, } \\
\text { ajusteurs et tailleresses }\end{array}$ & 35 livres & 50 livres \\
\hline $\begin{array}{l}\text { Élection des officiers du } \\
\text { Serment de France }\end{array}$ & 14 livres & 24 livres 10 sols \\
\hline
\end{tabular}

Après avoir acquitté les droits de réception, le monnayeur peut jouir d'importants privilèges, avant tout fiscaux.

\section{«Barriers, péagers, pontonniers, laissez passer les monnoyers ${ }^{50}$ ”}

Les privilèges, forts anciens, sont en effet particulièrement importants : ils apparaissent comme étant originellement une compensation à un travail précaire, contraignant et mal payé, source d'une nécessaire pluriactivité. Les confirmations des privilèges indiquent que ceux-ci, pour la Monnaie de Nantes, doivent remonter au moins à $1420^{51}$. Privilèges remon-

46. Une prospection dans la capitation de Nantes de 1762 a identifié un sieur Couillaud Dubreil " blettier et monnoyeur ", Arch. mun. de Nantes, CC 460, Rôle de capitation de Nantes pour l'année $1762, \mathrm{f}^{\circ} 113 \mathrm{r}^{\circ}$.

47. Arch. dép. de Loire-Atlantique, B 5354, Procès-verbal de nomination et d'élection d'un prévôt et lieutenant des monnoyeurs à la Monnoye de Nantes, 11 août 1764.

48. Arch. dép. de Loire-Atlantique, B 5221, Arrêt de la Cour des Monnaies du 23 décembre $1754, \mathrm{f}^{\circ} 61 \mathrm{r}^{\circ}-\mathrm{f}^{\circ} 64 \mathrm{r}^{\circ}$.

49. Arch. dép. de Loire-Atlantique, B 5222, Arrêt de la Cour des Monnaies du 17 mars $1779, \mathrm{f}^{\circ} 63 \mathrm{v}^{\circ}-\mathrm{f}^{\circ} 66 \mathrm{r}^{\circ}$.

50. Il s'agit de la formule inscrite sur une médaille qui, selon Raoul Toscan, est portée par les monnayeurs lors des déplacements sur la Loire, affirmant l'exemption des péages dont ils bénéficient : TosCAN, Raoul, L'Épopée des Mariniers de la Loire, Delayance, 1938, p. 76.

51. Arch. dép. de Loire-Atlantique, B 5275, Privilèges, franchises, libertez et exemptions des officiers, ouvriers et monnoyers de la Mônnoye, leurs concedez et octroyez par les Ducs de Bretagne, Roys de France, vérifiez de temps en temps par Arrests de la Cour de Parlement dudict Pays, Nantes, Pierre Doriov, Imprimerie ordinaire du Roy, 1619. Il s'agit d'une compilation des confirmations de privilèges du duc François II (1483) à Louis XIII (1612). Pour 
tant donc aux ducs de Bretagne, mais paraissant en tous points identiques aux autres hôtels de Monnaie du royaume.

En quoi consistent donc ces privilèges apparaissant si convoités au XVIII ${ }^{\mathrm{e}}$ siècle? Les officiers de la Monnaie et le personnel du Serment de France sont exempts « de tailles [fouages pour la Bretagne], taillons, subsides, aides, de tous droits de voierie, impositions, subventions, contributions, emprunts, fortifications, réparations, entrées de villes, péages, passages, de toutes levées ordinaires et extraordinaires, du logement de gens de guerre, de guet, garde des portes, sentinelles, tutelles, curatelles, dépôts, garde de biens de justice, commissions, corvées personnelles et autres de telle espèce que ce puisse être, garderies de marchand et autres charges personnelles, publiques et de police ${ }^{52}$ ". Ces privilèges sont donc d'abord fiscaux et sont un héritage médiéval : les monnayeurs ne sont pas exemptés des créations fiscales et de charges modernes. De cette liste, on retiendra bien entendu l'exemption des fouages : c'est cette exemption qui excite le plus la jalousie et celle dont se vantent en premier lieu les monnayeurs, comme nous l'avons vu avec Silvestre Chauvelon. On notera également l'exemption de la corvée et de la milice. Mais si les monnayeurs sont exemptés de la milice, ils ne le sont a priori pas du service des classes : nous verrons plus loin ce qu'il en est dans les faits.

À la liste de ces exemptions d'impositions, contributions et charges publiques, il faut ajouter un deuxième type de privilège : le privilège juridictionnel. La Cour des monnaies possède en effet " un droit exclusif et absolu de police et de juridiction, tant au civil qu'au criminel, sur les officiers et agents des monnaies pour tout ce qui se rapporte à l'exercice de leurs fonctions ${ }^{53}$ ". Elle agit en première instance avec appel à la chambre des Monnaies de Paris (depuis l'annexion de la Bretagne), en revanche les rapts, larcins et meurtres relèvent des tribunaux ordinaires ${ }^{54}$.

Comme nous l'avons déjà évoqué, ces privilèges ne peuvent manquer d'attirer la convoitise de tout un petit monde de grands notables nantais, dont on n'imagine pas un instant que le désir d'être reçu au Serment soit motivé par une passion pour la frappe au balancier. Dans une stratégie de recherche de noblesse, l'exemption fiscale des impositions roturières qu'apportent les privilèges de la Monnaie, c'est-à-dire un quasi-alignement fiscal sur la noblesse, ne peut qu'aiguiser l'appétit d'une bourgeoisie dont les portes de la Monnaie lui ont été ouvertes par les créations royales et lui res-

les privilèges octroyés en 1420 par le duc Jean V : Bigot, Alexis, Essai sur les monnaies du royaume et duché de Bretagne, Paris, Rollin, 1857, p. 376-379; LA NicolLIERE-TEIJEIRO, Stéphane de, Privilèges de la ville de Nantes, Nantes, Bibliophiles bretons, 1883, p. 56-57.

52. Arch. mun. de Nantes, HH 24, pièce 8, Lettres patentes du Roi en forme d'édit portant renouvellement et confirmation des privilèges des Monnoyeurs, ajusteurs et tailleresses du Serment de France et des officiers des Monnoies, Données à la Muette au mois d'octobre 1782, p. 2.

53. Costes, Henri, Les Institutions monétaires de la France avant et depuis 1789, Paris, Guillemin et $\mathrm{C}^{\mathrm{ie}}, 1885$, p. 178.

54. Manceron, Paul, op. cit., p. 5. 
tent ouvertes au XVIII ${ }^{\mathrm{e}}$ siècle par les très convoitées tailleresses ${ }^{55}$. Le but de ces privilèges est de pallier les contraintes inhérentes à l'activité du monnayage. Activité à temps partiel, aléatoire, où le monnayeur doit en théorie, à l'appel du prévôt, tout quitter, foyer ou travail, pour rejoindre l'atelier et ce, sous peine d'amende, le monnayage est en plus bien mal payé.

Il existe en fait différents types de revenus, tous modiques. Il y a d'abord le salaire du monnayage proprement dit que l'on appelle le "brassage ", le monnayeur peut également bénéficier de différents petits revenus complémentaires comme les droits perçus à l'occasion des réceptions pour les monnayeurs ayant surveillé l'épreuve, payés par l'apprenti sous le nom de « droit de cuissières ". Il existe aussi un fonds commun, administré par les prévôts : " les droits de boites ", alimenté par des apports personnels, annuels et obligatoires ainsi que par les amendes, le tout constituant une sorte de fonds de solidarité ${ }^{56}$. Concernant le salaire du monnayage proprement dit, celui-ci n'a pas varié entre 1696 et 1785 et il est le même pour les monnayeurs et les ajusteurs : 2 sols par chaque marc d'or et 1 sol par marc d'argent; après 1785, il passe à 3 sols par chaque marc d'or et 1 sol 6 deniers par marc d'argent ${ }^{57}$.

Les lettres patentes de Louis XVI soulignent que " sans la liberté qu'ils ont toujours eue de faire le commerce, la plupart de nos monnoyeurs seroient hors d'état de subister à cause de la modicité de leurs droits ${ }^{58}$ ".

55. Il n'est pas de notre propos d'étudier ce type de comportement parasitaire qui interfère pourtant avec notre population étudiée. Le phénomène est ancien : il remonte à la courtisanerie ducale, KERHERVE, Jean, op. cit., p. 203. Comme pour les tailleresses, ces individus n'apparaissent que dans le procès-verbal de leur réception ou les rôles de personnel. Parmi ces ouvriers fantomatiques, citons : un capitaine de navire (Arch. dép. de LoireAtlantique, B 5354, Réception de Michel Maugeais, monnayeur, 5 mai 1758); la fille d'un ancien juge consul de Nantes (Arch. dép. de Loire-Atlantique, B 5308, Réception de Marie Magdelineau, tailleresse, 7 juin 1712); la veuve d'un procureur du Roi des Eaux et Forêts (Arch. dép. de Loire-Atlantique, B 5361, Réception de Jeanne Forget, tailleresse, 30 janvier 1778); le " noble homme " Pierre-Etienne Robin de Blanche-Noë comme ouvrier ajusteur (Arch. dép. de Loire-Atlantique, B 5360, Réception du 4 avril 1775). Et que dire enfin des Richard, parmi lesquels on relève le nom de Georges Richard, l'un des grands négociants nantais de la seconde moitié du XVIII ${ }^{\mathrm{e}}$ siècle, reçu ajusteur le 13 mars 1745! (Arch. dép. de Loire-Atlantique, B 5344, Réception de Georges Richard, ajusteur, 13 mars 1745).

56. Planchenault, Adrien, op. cit., p. 90. Caisse commune de solidarité servant notamment au financement des nombreux procès auxquels doivent faire face les monnayeurs, sur lesquels nous reviendrons. C'est ainsi, par exemple, que Pierre Peillac qui s'était retrouvé malgré " [l'] exemption des monnoyeurs de toutes sortes de collectes " à faire " la collecte et l'amas des rentes de Bougon " à Bouguenais, est contraint de devoir payer 271 livres 11 sols et 4 deniers suite à son procès perdu au Présidial puis au Parlement. Il demande ainsi à ce que " le coffre des monnoyers et ouvriers de la Monnoye de cette ville " soit ouvert pour prendre la somme parce que la cause qu'il a défendu est " une affaire commune ". Le coffre est effectivement ouvert. Arch. dép. de Loire-Atlantique, B 5305, Requête de Pierre Peillac, 8 et 13 août 1709.

57. Arch. dép. de Loire-Atlantique, B 5285, Édit du Roi portant Règlement pour la fabrication des espèces, et augmentation des droits des Officiers des Monnoies, sur la conversion des matières d'Or et d'Argent, donné à Fontainebleau au mois de novembre 1785.

58. Arch. mun. de Nantes, HH 24, pièce VIII, Lettres patentes du Roi en forme d'édit portant renouvellement et confirmation des privilèges des Monnoyeurs, ajusteurs et tailleresses 
Dans une requête adressée au secrétaire d'État à la Marine, Pontchartrain, des pêcheurs-monnayeurs d'Indre devant faire face à la jalousie d'habitants qui a conduit à leur inscription sur les rôles " des soudes et fouages ", insistent sur la nécessité pour eux de pratiquer la pêche car « le travail de la monnoye ne produit pas des rétributions suffisantes pour faire subsister leurs familles ${ }^{59}$ ". Le monnayage, travail précaire, nécessite donc l'exercice d'une activité principale, les privilèges n'étant pas suffisants pour pallier le handicap salarial. À Nantes, les monnayeurs forment au XVIII ${ }^{\mathrm{e}}$ siècle une population d'abord rurale, dont la plus forte minorité est constituée de pêcheurs.

\section{Être pêcheur au sein du Serment de France}

Il s'agit à présent de saisir cette population de pêcheurs-monnayeurs, tant au niveau quantitatif que qualitatif, et s'efforcer d'approcher les interactions existant entre l'activité pêche et celle du monnayage.

\section{Des monnayeurs insulaires}

Différents états du personnel de l'atelier monétaire de Nantes ont été conservés, malheureusement, ils se trouvent répartis chronologiquement de façon très déséquilibrés, plusieurs rôles ayant été conservés pour les années 1680 et 1690 , mais aucun pour le XVIII ${ }^{\mathrm{e}}$ siècle sauf un pour 1728 . Sont donc retenues ici deux listes complètes, celle de 1697 et celle de 1728 , offrant une évolution sur le premier tiers du XVIII siècle $^{60}$.

Déterminer dans ces rôles lesquels de ces monnayeurs sont pêcheurs n'est pas une tâche facile compte tenu de l'état des sources. En effet, les sentences des réceptions à l'atelier monétaire ne mentionnent pas l'activité professionnelle des monnayeurs en dehors de l'atelier, il faut donc les recouper avec d'autres sources. Le problème pour Rezé, qui concentre l'essentiel des pêcheurs-monnayeurs, est que ni les sources fiscales, ni les registres paroissiaux pour la plus grande partie du siècle ne mentionnent les professions ${ }^{61}$. Il faut donc recouper systématiquement ces derniers avec les matricules des classes, les sources notariales et judiciaires, pour comp-

du Serment de France et des officiers des Monnoies, Données à la Muette au mois d'octobre 1782, p. 3.

59. Arch. dép. de Loire-Atlantique, B 5291, Requête de Jean et Gabriel Mottin, Joseph Saulny, Jean Loyen et Julien Richeux, " ouvriers et monnoyeurs de la monnoye de Nantes ", non datée, années 1690 .

60. Arch. dép. de Loire-Atlantique, B 5275, Liste des noms et surnoms des Maîtres ouvriers et Monnoyers de la Monnoye de Nantes et le lieu de leur résidence que Jan Peillac et Jan Coiffé, prévosts de la Confrérie de Saint-Louis déclarent mettre au greffe de la sénéchaussée de Nantes suivant les Règlements, 13 septembre 1697; Arch. mun. de Nantes, HH 25, pièce 37, Liste de monnoyeurs, ajusteurs, tailleresses et veuves de la monnoye de Nantes, 26 février 1728 .

61. Les rôles de la capitation, en revanche, précisent ceux des capités qui sont monnayeurs, compte tenu de leurs nombreuses exemptions fiscales, mais pas l'activité qu'ils pratiquent à côté du monnayage. 
tabiliser, mais non exhaustivement, cette population. Deux grands ensembles de pêcheurs-monnayeurs se dégagent de ces deux états : Indre et Rezé, paroisses riveraines de la Loire, à la porte aval de Nantes. Notons d'ailleurs qu'en exceptant les ouvriers bourgeois, les monnayeurs sont tous domiciliés à Nantes et dans les paroisses environnantes : pour pouvoir répondre à l'appel du prévôt, ils ne peuvent évidemment vivre trop éloignés de l'atelier. Ainsi, l'état du personnel de 1697 indique une tolérance de 6 lieues, distance qui a semble-t-il évolué au cours du temps ${ }^{62}$.

On observe pour Rezé une forte évolution dans le premier tiers du $\mathrm{XVIII}^{\mathrm{e}}$ siècle : si Rezé représente $25 \%$ des 73 monnayeurs et ajusteurs en 1697, cette localité en concentre $41 \%$ sur les 102 de la liste de 1728 passant ainsi de 18 individus à 43. Comment expliquer cette progression tant en valeur relative qu'absolue? Peut-être faut-il y voir des causes essentiellement démographiques.

\section{Figure 2 - Répartition géographique des 73 monnayeurs et ajusteurs inscrits sur le rôle du personnel de 1697}

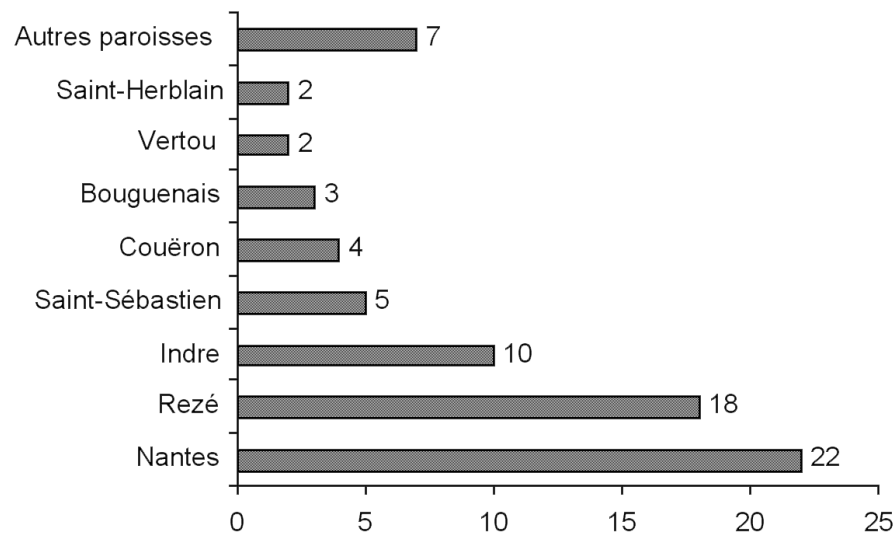

Parmi les monnayeurs et ajusteurs rezéens, quelle part peuvent représenter les pêcheurs? La capitation de 1740 donne une idée des proportions. Dans ce rôle figurent 45 monnayeurs. Sur ces 45,37 , soit 82 \% résident dans le " canton des Isles ", c'est-à-dire l'île des Chevaliers et l'île de Trentemoult, domaine des pêcheurs rezéens ${ }^{63}$. Seulement 8 résident ailleurs, 5 à Pont-

62. Arch. mun. de Nantes, HH 24, pièce IV, Lettre du dauphin Henri en qualité de duc de Bretagne confirmant les privilèges et exemptions d'impôts des Monnoyeurs de Nantes et de Rennes..., Fontainebleau, janvier 1545, donne pour condition la résidence des monnayeurs à moins de 3 lieues de l'atelier.

63. Les îles de Rezé sont constituées, de l'amont en aval : de l'île des Chevaliers avec les villages de la Haute-f̂le et de la Basse-Île, du hameau de Nortiouze et du village de Trentemoult, le plus peuplé, île dans les îles. Ces îles comptent 1025 habitants de plus de 12 ans en l'an IV, les pêcheurs représentant 88,8 \% des professions masculines et féminines déclarées, soit 371 individus. Arch. dép. de Loire-Atlantique, L 1642, État de la 
Rousseau et 3 dans le " canton des champs ${ }^{64}$ ". Un nombre - minimum de 34 pêcheurs, soit un peu plus de 75 \% des monnayeurs de Rezé, peut être avancé pour cette date.

\section{Figure 3 - Répartition géographique des 102 monnayeurs et ajusteurs inscrits sur le rôle du personnel de 1728}

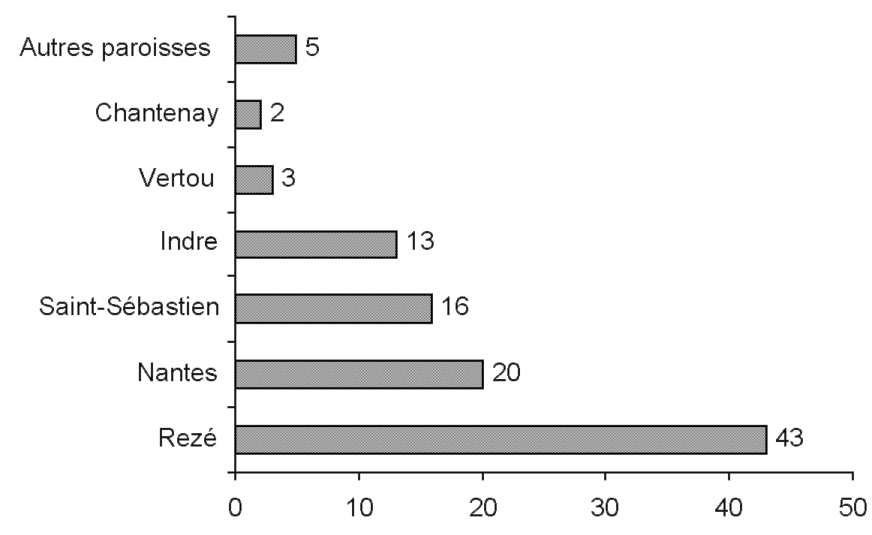

Les " îles " d'Indre constituent le deuxième ensemble de pêcheurs-monnayeurs ${ }^{65}$. La liste de 1697 révèle 10 noms de monnayeurs et ajusteurs soit $14 \%$ des effectifs, celle de 1728, 13 individus, soit $13 \%$. Contrairement à Rezé, le nombre reste stable au cours du premier tiers du XVIII ${ }^{\mathrm{e}}$ siècle, décline même un peu en valeur relative. Sur les 13 noms de 1728, le recoupement permet d'identifier un minimum de 11 pêcheurs. Des pêcheurs, cependant, qui ne font pas que pêcher et " monnayer", comme nous le verrons plus loin.

Rezé et Indre semblent donc concentrer la quasi-totalité des pêcheursmonnayeurs. Quelques pêcheurs isolés peuvent être localisés dans d'autres paroisses, comme Bouguenais, limitrophe à Rezé : par exemple, le seul monnayeur de la liste de 1728 habitant cette paroisse est effectivement un pêcheur ${ }^{66}$. Quoi qu'il en soit, en 1728 , les pêcheurs représentent au moins 40 \% des effectifs globaux du Serment de France, en exceptant les tailleresses.

population de la commune de Rezé en l'an IV de la République française une et indivisible. Tous les monnayeurs des îles identifiés sont domiciliés soit à Trentemoult, soit à la Haute-lle. 64. Arch. dép. de Loire-Atlantique, B 3507, Capitation de Rezé pour l'année 1740.

65. La paroisse d'Indre est formée de trois " îles " : Haute-Indre, Basse-Indre et Indret. Les monnayeurs sont domiciliés à Haute-Indre et à Basse-Indre, " île " la plus peuplée, située en aval de la précédente. Ces deux localités sont d'anciennes îles rattachées à la rive droite, mais gardant cependant au XVIII ${ }^{\mathrm{e}}$ siècle un certain caractère insulaire.

66. Arch. dép. de Loire-Atlantique, 120 J 192, Registres des matricules du quartier de Nantes, Matricules des matelots par classes, hors Nantes (1700-1732), f ${ }^{\circ} 84$, François Dejoie; Arch. dép. de Loire-Atlantique, B 5304, Réception de François Dejoie, ouvrier, 12 novembre 1707 . 
En l'absence de rôles du personnel postérieurs à 1728, il est difficile de suivre l'évolution de la place des pêcheurs par rapport aux autres monnayeurs au sein de l'atelier pour le reste du XvIII siècle. L'évolution des effectifs des pêcheurs peut cependant être approchée à partir des rôles de la capitation et du sondage effectué dans les sentences des réceptions à l'atelier ${ }^{67}$. En ce qui concerne Rezé, après la forte progression du premier tiers du siècle, les effectifs paraissent se stabiliser avant de fléchir dans le dernier tiers du siècle. En effet, si la capitation de 1740 comprend 45 monnayeurs dont 37 pour le canton des " isles ${ }^{68}$ ", le rôle de 1762 en mentionne 33 pour ce même canton des îles ${ }^{69}$. La capitation de 1789 , moins fiable ${ }^{70}$, confirmerait cette baisse puisqu'une petite vingtaine de monnayeurs, identifiés ou hypothétiques, habiteraient alors dans les îles ${ }^{71}$. Les sentences des réceptions à l'atelier monétaire témoignent du reflux : probablement moins de 10 pêcheurs sont reçus à la Monnaie dans les années $1780^{72}$.

Cette désaffection ne semble pas propre aux pêcheurs, et à l'atelier de Nantes, comme en témoigne l'arrêt du Conseil du Roi du 28 octobre 1755 dont on a déjà parlé et dont l'existence montre bien que l'on peine alors à recruter ${ }^{73}$. L'unification du montant des droits des réceptions en 1754, la (petite) hausse des salaires en 1785 , démontrent le même problème ${ }^{74}$. Peutêtre les privilèges deviennent-ils moins intéressants au regard de l'évolution conjoncturelle? Si, comme nous le verrons, les contestations des privilèges des monnayeurs, à l'échelle locale, sont incessantes et nullement propres au XVIII ${ }^{\mathrm{e}}$ siècle, ces dernières vont trouver dans ce siècle un appui venu du " haut ». La seconde moitié du siècle est marquée en effet par une législation grignotant peu à peu les privilèges sur fond de montée du libéralisme traduite notamment par les campagnes anti-corporatistes menées par les physiocrates. Malgré le statut très particulier de la corporation des monnayeurs, unique et " abrité " par une enveloppe juridictionnelle, la Cour des Monnaies, la corporation n'échappe manifestement pas à la remise en cause générale de l'organisation du travail, même si elle n'est pas concernée par la suppression puis " réorganisation " des corporations en 1776-1777 ${ }^{75}$. Ainsi, si l'édit de 1760 confirme le privilège d'exemption de la taille, celui-ci, comme

67. Seul un dépouillement exhaustif des sentences des réceptions à l'atelier monétaire, recoupé avec les registres paroissiaux, permettrait de bien saisir l'évolution des effectifs.

68. Arch. dép. de Loire-Atlantique, B 3507, Rôle de capitation de Rezé pour l'année 1740.

69. Arch. mun. de Rezé, CC 8, Rôle de capitation de Rezé pour l'année 1762.

70. Les monnayeurs n'y sont pas systématiquement mentionnés, au contraire des rôles précédemment utilisés.

71. Arch. dép. de Loire-Atlantique, B 3531, Rôle de capitation de Rezé pour l'année 1789.

72. Arch. dép. de Loire-Atlantique, B 5188 -B 5189, B 5363-B 5369.

73. Arch. dép. de Loire-Atlantique, B 5282, Arrêt du Conseil du Roi « qui ordonne que tous ceux qui ont droit aux places de Monnoyeurs... ", 28 octobre 1755.

74. Arch. dép. de Loire-Atlantique, B 5221, Arrêt de la Cour des Monnaies du 23 décembre 1754, $\mathrm{f}^{\circ} 61 \mathrm{r}^{\circ}-\mathrm{f}^{\circ} 64 \mathrm{r}^{\circ}$; B 5285, Édit du Roi “ portant Règlement pour la fabrication des espèces... ", donné à Fontainebleau au mois de novembre 1785 .

75. Kaplan, Steven, La fin des Corporations, Paris, Fayard, 2001, 740 p. Cette étude ne traite pas du cas particulier des monnayeurs. 
l'ensemble des privilèges, est désormais subordonné à une justification qui devra être faite chaque année par le monnayeur du service effectué à la Monnaie $^{76}$. C'est une nouveauté : les privilèges ne sont ainsi plus acquis à vie, ils sont désormais, en théorie, renouvelables chaque année en fonction du travail effectué. Un deuxième édit en juillet 1766 restreint le privilège d'exemption de la taille d'exploitation au clergé, à la noblesse et aux officiers pourvus de charges anoblissantes, excluant donc les monnayeurs qui perdent là un privilège dont ils avaient toujours bénéficié ${ }^{77}$. Enfin, en 1772, par souci d'économie, un édit du Roi supprime treize Hôtels des Monnaies sur les trente existants, dont celui de Rennes, annulant de fait les privilèges de leurs ouvriers : c'est toute la corporation qui s'en trouve considérablement affaiblie ${ }^{78}$. Le coup de grâce vient avec la Révolution : les ajusteurs et tailleresses disparaissent avec la loi du 20 septembre 1791, les monnayeurs, eux, bénéficient d'un sursis jusqu'au 18 août 1792 .

Des privilèges donc menacés, une réglementation plus rigide liée à des salaires toujours dérisoires, peuvent avoir décidé les pêcheurs de s'éloigner peu à peu d'une Monnaie, sans doute quelque peu atone, dans la seconde moitié du XVIII ${ }^{\mathrm{e}}$ siècle; la prospérité du port de Nantes, dont les pêcheurs, surtout de Rezé, savent tirer profit, offrant de quoi compenser largement des privilèges fiscaux fragilisés. Le fait est là : le passage générationnel entre pêcheurs à l'atelier monétaire n'apparaît plus systématique dans les dernières années de l'Ancien Régime ${ }^{79}$. À la veille de la Révolution, c'est une page vieille de plusieurs siècles qui s'apprête à se tourner, celle d'une population qui aura concilié pêche et monnayage.

\section{Monnayeurs sur Loire et monnayeurs sur mer}

Trois principales sources permettent d'approcher l'activité de pêche des monnayeurs : les congés délivrés par l'amirauté de Nantes pour les sorties en mer, les sources accidentelles (déclarations d'avaries, procédures judiciaires) et les inventaires (après décès ou effectués à l'occasion d'un remariage), ces derniers permettant, en principe, d'identifier les types de filets utilisés et donc les types de pêches pratiqués ${ }^{80}$.

76. Arch. dép. de Loire-Atlantique, B 5283, Arrêt du Conseil d'État du 5 février 1760.

77. Arch. mun. de Nantes, HH 24, pièce 8, Lettres patentes du Roi en forme d'édit portant renouvellement et confirmation des privilèges des Monnoyeurs, ajusteurs et tailleresses du Serment de France et des officiers des Monnoies, Données à la Muette au mois d'octobre 1782, p. 2.

78. Arch. dép. de Loire-Atlantique, B 5284, Edit du Roi portant suppression des Monnoies..., février 1772.

79. À l'exemple de Sébastien Ollive, fils aîné de Sébastien Ollive et Jeanne Allaire (voir tableau en annexe), 39 ans en 1789 et qui ne s'est pas fait recevoir. Idem pour son frère cadet Jean Ollive.

80. On notera sur ce point que si les inventaires de Rezé, qui sont dressés par un greffier du présidial, détaillent presque toujours les types de filets, cela est très rarement le cas pour Indre, où ils sont dressés par un notaire seigneurial. 
Les pêcheurs monnayeurs des "Isles " de Rezé se situent dans ce qui constitue au XVIII ${ }^{\mathrm{e}}$ siècle le foyer le plus important et le plus actif de la pêche côtière et fluviale de l'Amirauté de Nantes. Citons ici l'inspecteur des pêches Le Masson du Parc, en inspection dans l'Amirauté en 1728 :

"Les pêcheurs de Trentemou sont avec ceux de l'isle des Chevaliers leurs voisins les plus fameux ou pour mieux dire les plus expérimentés des pêcheurs de la Loire et qui fournissent seuls tout le poisson de mer qui se consomme à Nantes [Le Masson du Parc a sans doute ici une légère tendance à l'exagération...], et ils sont même eux seuls en plus grand nombre que tous les autres pescheurs ensemble qui se trouvent le long des costes du ressort de cette amirauté quoique d'une grande étendue [ce qui n'est pas là exagéré $]^{81}$."

Quelques décennies plus tard, Duhamel du Monceau confirme la bonne réputation de ces pêcheurs :

"Les pêcheurs de Trentemou et ceux de l'isle des Chevaliers passent pour les plus habiles du canton; quelques uns vont à la mer et s'éloignent jusqu'en Poitou et à Belle-Isle; d'autres ne remontent la rivière que jusqu'au pont de Nantes ${ }^{82}$."

La flottille de pêche rezéenne, qui constitue l'essentiel de la flotte de pêche fluviale et côtière de Nantes, est composée en 1728, selon Le Masson du Parc, de 100 barges dont le tiers environ prendrait la mer, proportion allant crescendo au cours du siècle ${ }^{83}$.

Les pêcheurs de Rezé embarquent dans les barges à trois pour la pêche en mer et deux pour la pêche fluviale ${ }^{84}$; pour la pêche en Loire, ils se font même " souvent aider par des femmes et des filles ${ }^{85}$ ". La principale activité en Loire, c'est la pêche aux poissons migrateurs. Les pêcheurs de Rezé s'appuient pour cette dernière sur un privilège de pêche dans l'estuaire de la Loire octroyé en 1397 par le duc Jean IV ${ }^{86}$. Jean Dejoie, pêcheur-mon-

81. Arch. dép. de Loire-Atlantique, $120 \mathrm{~J}$ 485, Procès-verbal de la visite concernant la Pesche le long des Costes du ressort de l'amirauté de Nantes par Le Masson du Parc, le 16 septembre 1728 .

82. Duhamel Du Monceau, Henri-Louis, Traité général des pesches, et histoire des poissons qu'elles fournissent, tant pour la subsistance des hommes que pour plusieurs autres usages qui ont rapport aux arts et au commerce, réedit en fac-sim. de l'édition de 1772, Paris, CME, 1998, p. 259.

83. Arch. dép. de Loire-Atlantique, $120 \mathrm{~J}$ 485, Procès-verbal de la visite concernant la Pesche..., op. cit. Des apostilles, postérieures à 1768, font état d'une flottille de pêche de 110 barges. Toutes celles de Trentemoult, au nombre de 60, prendraient alors la mer. La barge des pêcheurs de Rezé est une petite barque à fond plat, sans quille, à voile carrée et à avirons. Rarement supérieures à un tonneau durant les deux premiers tiers du XVIII ${ }^{\mathrm{e}}$ siècle, ces barges commencent à gagner quelques tonneaux supplémentaires dans le dernier tiers du siècle, parallèlement à une activité maritime pêche/cabotage plus soutenue.

84. Idem.

85. DuHAMEl Du MonCEAu, Henri-Louis, op. cit., p. 259; également observé par Le Masson du Parc, Arch. dép. de Loire-Atlantique, 120 J 485, Procès-verbal de la visite concernant la Pesche..., op. cit.

86. Bibl. mun. de Nantes, 85198/C65, Mémoire pour les pêcheurs et habitans des Isles de Trantemoult, d'Ortiouze, des Chevaliers et Haute-Isle, Paroisse de Saint-Pierre de Rezé, 
nayeur de l'île de Trentemoult, possède ainsi des sédors, filets servant à la pêche aux saumons et aloses ${ }^{87}$ et des lampresses servant, comme le nom l'indique, à la pêche de la lamproie " qui est considérable ${ }^{88}$ ". Pêcheurs d'estuaire, certains bargers pratiquent également une pêche côtière, qui s'inscrit ainsi dans la prolongation du finage fluvial. Elle est de deux types : une pêche aux poissons frais le long des côtes de Bretagne et Poitou et une pêche aux harengs, cette dernière s'effectuant principalement en baie de Mesquer ${ }^{89}$; ainsi, en plus de ses filets pour la pêche fluviale, Jean Dejoie possède des filets à harang ${ }^{90}$. Des pêcheurs-monnayeurs sont présents dans les registres de congés de l'Amirauté de Nantes, congés demandés pour aller faire « la pesche au poisson frais le long des costes de Bretagne». Par exemple, en octobre 1722, on retrouve notre maître monnayeur de Trentemoult Silvestre Chauvelon à bord de sa barge La Chauvelone ${ }^{91}$, ou encore le maître monnayeur Silvestre Peillac en juin $1730{ }^{92}$. Ils n'apparaissent guère nombreux mais demandent-ils tous des congés pour sortir en mer? Des pêcheurs pouvant aussi se faire caboteurs, tant ces deux activités se lient facilement. Ainsi, en 1757, Sébastien Ollive, pêcheur-monnayeur de l'île des Chevaliers, cabote de la sardine entre Le Croisic et Nantes, avec sa barge La Thérèse ${ }^{93}$.

Le profil, si commun ailleurs, du pêcheur-paysan, correspond-il aux pêcheurs de Rezé? L'ensemble insulaire rézéen, îles basses inondées par la Loire une partie de l'année, ne semble guère propice au développement d'activités agricoles considérables. Lors de leur procès contre la ville de Nantes, les pêcheurs se défendent d'ailleurs d'exercer une autre activité que la pêche. Pour eux, les droits de pêche dont ils bénéficient : «Font subsister leurs familles enfermées dans des Isles où elles n'ont pas le secours

diocèse de Nantes, contre les Maires et Échevins de la ville et Communauté de Nantes, 1745 , p. 10; pour les privilèges : JonEs, Michael, Recueil des actes de Jean IV, duc de Bretagne, Tome II (1383-1399), Paris, Klincksieck, 1983, p. 666-668.

87. ou " 7 dorts " (= maille de 7 doigts). Le Masson du Parc indique que ces filets peuvent également être utilisés en mer, Arch. dép. de Loire-Atlantique, $120 \mathrm{~J}$ 485, Procès-verbal de la visite concernant la Pesche..., op. cit.

88. Idem.

89. DuHAmel du Monceau, Henri-Louis, op. cit., p. 374.

90. Arch. dép. de Loire-Atlantique, B 6899/2, Inventaire Jean Dejoie, 13 mai 1763.

91. Arch. dép. de Loire-Atlantique, B 4717, Enregistrement des congés de sorties pour la province de Bretagne, 15 octobre $1722, \mathrm{f}^{\circ} 151 \mathrm{v}^{\circ}$.

92. Arch. dép. de Loire-Atlantique, B 4661, Enregistrement des rapports des capitaines au long cours et au cabotage, des maîtres de barques venant de la province et hors province, 9 juin $1730, \mathrm{f}^{\circ} 72 \mathrm{r}^{\circ}$. Concernant le dit Silvestre Peillac, on ne peut manquer au passage, pour l'anecdote, d'indiquer ce qu'a relevé le marquis des Granges de Surgères : " dans la collection d'argenterie ancienne de M. Paul Eudel, vendu à Paris les 25-26 avril 1884, on remarque le $\mathrm{n}^{\circ} 130$ du catalogue : Tasse à déguster le vin, de forme ronde et surbaissée, avec armes de France et de Navarre et effigie de Louis XV; date de 1726 et l'inscription: Sylvestre Peillac, maître monnoyeur ", GRANGES DE SURGERES, Marquis des, op. cit., p. 368, maître monnayeur et pêcheur de hareng pourrait-on ajouter!

93. Arch. dép. de Loire-Atlantique, B 4616, Enregistrement des rapports des capitaines au cabotage et maîtres de barques de la province de Bretagne, 26 septembre $1757, \mathrm{f}^{\circ} 38 \mathrm{v}^{\circ}$. 
que d'autres occupations utiles leur donneroient ${ }^{94}$. "Sur ce point, les sources dépouillées ne permettent pas d'infirmer leur propos ${ }^{95}$.

Les pêcheurs d'Indre présentent un profil plus polyvalent que les pêcheurs de Rezé. Ils peuvent en effet conjuguer à la pêche deux autres activités importantes sur la Loire. La première est le voiturage ${ }^{96}$, consistant d'abord au transport des passagers au sein du complexe portuaire, activité qu'ils dominent; ils peuvent également participer au transbordement des marchandises entre Nantes et ses avants-ports, leurs petites barges affrontant alors une difficile et conflictuelle concurrence avec l'armada des gabares nantaises. L'autre activité est le pilotage. Louis Oresve, par exemple, pêcheur, est reçu monnayeur le 5 septembre 1719, ce qui ne l'empêche pas d'être reçu le mois suivant pilote lamaneur. Il deviendra maître monnayeur le 7 avril $1725^{97}$. Il n'est d'ailleurs pas le seul pêcheur monnayeur à avoir été reçu pilote ce jour-là : c'est également le cas d'Yves Saulny ${ }^{98}$. Le fait n'est pas étonnant : station de pilotage de "l'intérieur", Indre est le principal foyer de pilotes lamaneurs de l'Amirauté de Nantes avec Saint-Nazaire. Ces pilotes de l'intérieur de la Rivière sont essentiellement recrutés parmi les pêcheurs, ces derniers étant par leurs activités les plus compétents pour identifier les bancs de sable de la Loire ${ }^{99}$.

Ce profil de monnayeur pilote semble avoir été assez rare au $\mathrm{XVIII}^{\mathrm{e}}$ siècle, non négligeable cependant, puisqu'un recoupement entre les réceptions à l'atelier monétaire et celles des pilotes à l'Amirauté en a identifié onze, biactivité que l'on retrouve d'ailleurs déclarée dans les registres paroissiaux ${ }^{100}$. Comptabilité qui ne tient bien sûr pas compte des pilotes

94. Bibl. mun. de Nantes, 85198/C65, Mémoire pour les pêcheurs et habitans des Isles de Trantemoult..., op. cit., p. 39.

95. L'activité agricole paraît se limiter au jardinage et à la culture de quelques oseraies. Le bétail est absent de la plupart des inventaires. Les terres possédées en dehors des îles sont affermées.

96. C'est généralement le terme de "barger » qui est utilisé dans les sources pour désigner une activité de voiturage, utilisation guère satisfaisante car prêtant à confusion.

97. GRANGES DE SuRgeres, Marquis des, op. cit., p. 361 ; Arch. dép. de Loire-Atlantique, B 4484, f ${ }^{\circ}$ 105, Réception de Louis Oresve, pilote lamaneur, 19 octobre 1719.

98. Arch. dép. de Loire-Atlantique, B 4484, f ${ }^{\circ} 105$, Réception d’Yves Saulny, pilote lamaneur, 19 octobre 1719.

99. Pour citer les officiers de l'Amirauté : " ce qui paroist le plus convenir pour estre reçus pilotes sont les pescheurs qui sont chaque jour sur les écueils ", Arch. dép. de Loire-Atlantique, C 878, Observations des officiers de l'Amirauté de Nantes (1750-1752), $\mathrm{f}^{\circ} 210 \mathrm{r}^{\circ}$.

100. Les registres paroissiaux d'Indre, plus bavards que ceux de Rezé, rendent en effet généralement compte de cette biactivité. Pour reprendre quelques exemples cités par Hubert Le Bastard : le 19 septembre 1733, à son décès, Louis Oresve est qualifié de "Maistre monoyeur de Nantes et Maistre pilote sur la rivière de Loire "; au baptême de son fils, le 17 septembre 1748, Pierre Mocquard est " Monoyer et pilote de la rivière "; au baptême de son fils, le 26 avril 1759, Pierre Saulny est qualifié de « $\mathrm{M}^{\mathrm{e}}$ monnoyeur à la Monnoye de Nantes et $\mathrm{M}^{\mathrm{e}}$ pilote sur la rivière de Loire "; à son enterrement, le 16 février 1760, Yves Saulny est quant à lui « $\mathrm{M}^{\mathrm{e}}$ pilote de la manœuvre et Monnoyeur à la Monnoye de Nantes ", LE BASTARD, Hubert, op. cit., p. 36; Arch. dép. de Loire-Atlantique, 1 Mi EC 440, Registres paroissiaux d'Indre, bapt., mar., sépult., 1718-1720,1726-1762. 
non-jurés, identifiables, ponctuellement, par les sources judiciaires. Ces monnayeurs devenus pilotes conservent-ils leur activité première c'est-àdire la pêche? On peut le penser dans la mesure où le pilotage n'est qu'une activité aléatoire à temps partiel. Les registres paroissiaux, les actes notariés, mais surtout les sources judiciaires peuvent rendre compte parfois de cette multiactivité. Ainsi, dans une déposition judiciaire, Yves Saulny est qualifié de " $\mathrm{M}^{\mathrm{e}}$ pilote, pescheur, barger et monnoyeur en la cour des monnoyes de Nantes ${ }^{101}$ ". L'activité pêche des pilotes et voituriers monnayeurs peut également être identifiée par la présence de filets dans les inventaires de ces derniers. L'inventaire avant remariage de Louis-Luc Oresve, pilote et monnayeur, mentionne ainsi des " filets pour la pêche ", prisés 18 livres ${ }^{102}$. Notons que le profil de pilote-monnayeur n'est pas une exclusivité des pêcheurs d'Indre. Sur les onze cas identifiés, trois concernent en effet des pêcheurs de Rezé, tous reçus pilotes dans la seconde moitié du XVIII ${ }^{\mathrm{e}}$ siècle.

Ajoutons enfin l'existence du profil de monnayeur-charpentier, de barge bien sûr. René Dejoie de l'île des Chevaliers, reçu monnayeur en 1708, est ainsi signalé comme étant également "charpentier et pescheur ${ }^{103}$ ". Et que dire de Joseph Mocquard, charpentier de barge, monnayeur et notaire ${ }^{104}$ ?

\section{Concilier la barge et le balancier}

Deux activités aux contraintes potentiellement fortes, deux statuts devant le Roi : celui du matelot et celui du monnayeur. Comment la cohabitation se passe t-elle?

\section{Bargers avant tout}

Une activité de pêche, parfois de voiturage ou pilotage, pouvant donc se traduire par des "sorties " de quelques jours est exercée en même temps qu'une activité de monnayage où le monnayeur doit pouvoir répondre à tout moment à son prévôt, les monnayeurs ayant à cet appel "l'obligation [...] de tout quitter, même leurs propres affaires, pour le service de la

101. Arch. dép. de Loire-Atlantique, B 4778, Enquête faite à requête de Jean et Jacques Mainguet, 5 septembre 1733.

102. Arch. dép. de Loire-Atlantique, B 11493, Inventaire Louis-Luc Oresve, 2 octobre 1759. 103. Arch. dép. de Loire-Atlantique, 120 J 192, Registre des matricules du quartier de Nantes, Matricules des matelots par classes, hors Nantes (1700-1732), Rezé, f $^{\circ} 54$.

104. Profil n'apparaissant que par recoupement, ce dernier préférant se déclarer monnayeur et/ou notaire, plus rarement charpentier. Pour prendre l'exemple des actes de décès de deux de ses fils : dans son acte de décès du $1^{\text {er }}$ avril 1833, René-Donatien Mocquard " charpentier de barge " est dit fils de feu Joseph Mocquard " notaire " et de feue Marguerite-Anne Raguideau; dans son acte de décès du 9 janvier 1840, Jean-Simon Mocquard, " ancien monnoyeur et tonnelier " est dit fils de feu Joseph Mocquard " monnoyeur et charpentier " et de Marguerite-Anne Raguideau. Arch. dép. de Loire-Atlantique, État civil d'Indre, 3 E 74/32. 
Monnoie $^{105}$ ". Comment les contraintes de l'une se sont accommodées des contraintes de l'autre?

Pour le général de paroisse d'Indre, l'affaire est entendue : [les monnayeurs] ont été et sont encore pescheurs, bargers ou voituriers par eau, pilotes et d'autres vacations qui leur procurent tous les jours des émoluments bien plus lucratifs que ceux de la monoye où ils ne vont jamais ${ }^{106}$. Jugement partial dont la radicalité ne peut être que suspecte mais qui doit être discuté. Effectivement, conjuguer deux activités, dont l'une, le monnayage, n'est intéressante que pour les privilèges et non le travail en tant que tel, très mal payé, la tentation est sans doute grande de privilégier systématiquement son activité principale, en l'occurrence son activité fluviale et maritime, au détriment de l'autre, même si cette dernière est à temps partiel et aléatoire. Deux types de sources permettent d'approcher l'attitude des monnayeurs vis-à-vis de leur devoir à l'atelier monétaire. Le premier renseigne sur l'assiduité des monnayeurs aux grands événements de l'atelier monétaire : ce sont les procès-verbaux des réceptions et des élections, mentionnant, mais pas systématiquement, les noms des monnayeurs présents, ces derniers signant de plus les procès-verbaux s'ils en ont la capacité. Un deuxième type de sources permet lui de se faire une idée de l'assiduité des monnayeurs au travail : il s'agit principalement des amendes, consignées dans les registres du greffe, et des procédures judiciaires.

En restant prudent, car il faudrait pouvoir procéder à un recoupement sur l'ensemble des élections, on peut estimer que la participation des bargers à ces dernières semble assez aléatoire et paraît logiquement en partie conditionnée par l'importance de l'élection. En 1745, par exemple, pour l'élection du prévôt des monnayeurs André Ertaud, les maîtres monnayeurs de Rezé sont pratiquement tous présents : ils sont au nombre de 28 sur les 36 personnes présentes. La participation de ces derniers est en revanche beaucoup plus modeste cinq ans plus tard lors de l'élection du nouveau lieutenant des monnayeurs, mais la proportion reste identique : 14 sur les 19 maîtres présents ${ }^{107}$. On peut procéder également à un recoupement entre la liste du personnel de l'atelier de 1728 et les réceptions de cette même année. Des trois réceptions conservées, malheureusement une seule mentionne les noms des monnayeurs, ou plutôt ici des ajusteurs, présents. La prudence est donc ici également de rigueur. Lors de la réception de Guillaume Aubin en tant que maître ajusteur ${ }^{108}$, ce sont ainsi 27 ajusteurs

\footnotetext{
105. Arch. mun. de Nantes, HH 24, pièce 8, Lettres patentes du Roi en forme d'édit portant renouvellement et confirmation des privilèges des Monnoyeurs, ajusteurs et tailleresses du Serment de France et des officiers des Monnoies, données à la Muette au mois d'octobre 1782, p. 3.

106. Arch. mun. d'Indre, CC 3, Défenses que fournit au siège Présidial de Nantes le général de la paroisse d'Indre, 13 février 1750.

107. Arch. dép. de Loire-Atlantique, B 5230, Élection d'André Ertaud, prévôt des monnayeurs, 14 décembre $1745, \mathrm{f}^{\circ} 23$; élection de Guillaume Peillac, lieutenant des monnayeurs, 14 février $1750, \mathrm{f}^{\circ} 32 \mathrm{v}^{\circ}$.

108. Arch. dép. de Loire-Atlantique, B 5327, Réception de Guillaume Aubin, maître ajusteur, 9 septembre 1728 .
} 
qui sont présents sur les 42 (bourgeois inclus) que compte alors l'atelier. Pour Rezé, 15 ajusteurs sur les 18 de la liste sont présents. Une assiduité qui serait donc ici assez remarquable, resterait à savoir si elle est ou non exceptionnelle; il faudrait également pouvoir évaluer son évolution dans le temps. Au moins cet exemple a-t-il le mérite de montrer qu'au moins de temps à autre, les monnayeurs de chaque chambre peuvent être presque tous réunis à l'atelier.

Si les monnayeurs paraissent d'une assiduité relative aux grands évènements de l'atelier, qu'en est-il pour le travail à la Monnaie? Payés modestement en fonction du marc frappé, les monnayeurs ne peuvent pas être très motivés à venir fabriquer des pièces de faible valeur. C'est ainsi que le 27 mars 1714, sept ajusteurs de Rezé, dont cinq pêcheurs, avertis comme de coutume par le lieutenant des ajusteurs, se présentent à l'atelier " entre les huit heures et neuf heures du matin ", mais une fois montés dans la chambre des ajusteurs, " ayant vu que le travail estoit d'ajuster des pièces de dix sols ou dixième d'escu, [ils] se sont retirés ". Trois d'entre eux sont condamnés à payer 30 sols d'amende et les quatre autres 3 livres, avec défense "à eux et à tous autres de s'absenter à l'avenir du travail qui se présentera en cette monoye " sous peine d'être " deschus de leurs fonctions et privilèges et d'estre rayé du cathalogue des ouvriers ajusteurs de cette monoye ${ }^{109}$ ". Punition pas vraiment exemplaire puisque quelques semaines plus tard, le 22 juin 1714, ce sont cette fois treize autres ajusteurs, tous bargers, qui refusent de venir ajuster les pièces de 10 sols ${ }^{110}$. Ils sont condamnés à payer chacun une amende de 10 livres et à être rayés du rôle des ouvriers en cas de récidive, menace, on le voit, guère appliquée ${ }^{111}$.

Mais l'absentéisme peut se manifester autrement. C'est ici qu'entre en scène une population pratiquement absente des sources et pourtant fondamentale à la bonne marche de l'atelier : les journaliers. Leur présence a deux origines : la première peut venir d'une embauche faite par le directeur de la Monnaie pour ajuster les effectifs, les réceptions au sein de l'atelier monétaire étant déterminées, comme on l'a vu, par la filiation et non l'offre de travail; la deuxième origine vient d'une embauche par les monnayeurs eux-mêmes. En effet, les monnayeurs qui en ont les moyens peuvent se faire remplacer et ce recours aux commis n'est pas, loin s'en faut, un monopole de la bourgeoisie fantomatique du monnayage. Ainsi en 1701, Mathurin Ertaud se plaint que « dans le monnoyage, les monnoyeurs y mettent des gens de journées et qu'il y a des monnoyeurs qui n'ont point encore entré en la monnoye pour y travailler ", dénonçant en passant un Peillac et autres monnayeurs qui " amènent journellement des journaliers qui ne sont de la Monnoye travailler au monnoyage ${ }^{112}$ ". Autre témoignage de la présence de

109. Arch. dép. de Loire-Atlantique, B 5310, Audience du 27 mars 1714.

110. Arch. dép. de Loire-Atlantique, B 5327, Liste des ouvriers ajusteurs qui ont refusé de venir travailler à ajuster des pièces de $10 \mathrm{~s}$. à la monoye de Nantes le 22 juin 1714. Trois de ces bargers sont de l'île des Chevaliers, cinq de Trentemoult et cinq autres d'Indre. 111. Arch. dép. de Loire-Atlantique, B 5310, Sentence du 22 juin 1714.

112. Arch. dép. de Loire-Atlantique, B 5301, Requête de Mathurin Ertaud, 5 décembre 1701. 
ces journaliers : en 1708, suite à une plainte verbale « des nommées Renaud, Abinais et consorts journaliers pris pour le travail de cette monoye " parce que l'on a " omis " de les payer, "les nommés Ertaud, Peillac et autres consorts ", maîtres ajusteurs qui les ont embauchés, sont condamnés à verser à ces journaliers solidairement la somme de 500 livres ${ }^{113}$.

S'il est difficile de savoir si ces agissements sont généralisés, car les sources sont rares, un arrêt de la Cour des Monnaies en 1730 fait état " d'une grande négligence pour le service de quelques-uns des hôtels des monnoyes de manière que le travail en est souvent retardé, se reposant les uns sur les autres pour faire ce travail, en sorte que dans beaucoup d'endroits il n'y en a qu'un très petit nombre qu'y satisfassent; que très souvent il n'y a qu'un monnoyeur au monnoyage ${ }^{114}$... ". Si l'absentéisme généralisé évoqué par le général de paroisse d'Indre ne vaut guère que pour la bourgeoisie ou les tailleresses, un certain laxisme semble donc bien caractériser l'attitude des bargers vis-à-vis de leur devoir à l'atelier. Si on tient compte, de plus, des périodes d'inactivité de l'atelier, parfois fort longues, des pêcheurs, tout en étant monnayeurs, peuvent donc sans grand souci cumuler les activités et s'afficher également pilotes, voituriers ou encore charpentiers.

\section{Servir le Roi en sa Monnaie ou servir le Roi sur les mers}

Population maritime et fluviale, les pêcheurs monnayeurs sont inscrits aux registres-matricules des classes afin, en théorie, de servir le Roi sur sa marine; mais ils doivent aussi le servir en sa Monnaie : comment ces deux devoirs cohabitent-ils?

Lors de la mise en place du système des classes, à la fin du XVII ${ }^{\mathrm{e}}$ siècle, les pêcheurs-monnayeurs ont certainement essayé de s'y soustraire, en faisant pression sur leur hiérarchie, comme tend à le montrer une ordonnance du 30 mai $1696^{115}$. En effet dans cette ordonnance, il est fait état de quelques frictions entre le commissaire de la Marine à Nantes et le directeur de la Monnaie. La cause en est que le commissaire a inséré " quelques particuliers ", qui se trouvent malencontreusement être des monnayeurs, "dans le rôle des classes des matelots". Pour le directeur de la Monnaie, ces particuliers sont " exempts de servir sur les vaisseaux de Sa Majesté attendu qu'ils sont ouvriers de la monnoye ", ce que ne précisent aucunement, et pour cause, les diverses confirmations de privilèges. "Voulant faire cesser ces contestations et rendre l'état de cette sorte de gens certain ", le statut des monnayeurs vis-vis du service des classes est donc fixé au bénéfice du commissaire de la Marine. Les monnayeurs ne sont en effet exemptés du service des classes que s'ils renoncent à la qualité de matelot. Il est ainsi

113. Arch. dép. de Loire-Atlantique, B 5216, Audience du 23 mars 1708, $\mathrm{f}^{\circ} 46 \mathrm{v}^{\circ}-\mathrm{f}^{\circ} 47 \mathrm{r}^{\circ}$. 114. Arch. dép. de Loire-Atlantique, B 5227, Arrest de la cour des monnoyes portant règlement pour les monnoyeurs, ajusteurs et tailleresses, 22 novembre 1730.

115. Arch. dép. de Loire-Atlantique, B 5293. 
ordonné que " dans trois mois à commencer du jour de la publication de la présente ordonnance, ces particuliers et tous autres qui sont dans le mesme cas, déclareront par devant le sieur de Richebourg commissaire ordinaire de la Marine au département de Nantes, s'ils veulent renoncer à la qualité de matelot auquel cas il ne leur sera pas permis d'en faire aucune fonction, pas mesme de pescher dans la Rivière, n'y de passer des gens d'un lieu à l'autre de la dite Rivière, après laquelle déclaration, Sa Majesté veut que leurs noms soient rayés du rôle des classes ". Les effectifs de bargers monnayeurs de la première moitié $d u$ XVIII ${ }^{\mathrm{e}}$ siècle montrent que cette déclaration d'abandon du métier de " matelot " n'a sans doute pas connu un grand succès.

Mais les monnayeurs ne désarment manifestement pas. En 1701, on retrouve la trace d'une lettre du Secrétaire d'État à la Marine, Pontchartrain ${ }^{116}$; ce dernier, répondant à une nouvelle requête du directeur de la Monnaie, rappelle l'obligation des monnayeurs de se conformer à l'ordonnance de 1696. Un quart de siècle plus tard, en 1725, une nouvelle ordonnance est publiée, réaffirmant l'ordonnance de $1696^{117}$. Cette réaffirmation a pour objet de " prévenir les constestations qui pourroient arriver [...] entre le Sieur Bigot de la Mothe Commissaire général de la Marine, Ordonnateur à Nantes, et le Directeur de la Monnoye en la même ville, comme il est desja arrivé dans le temps que la dite Ordonnance [celle de 1696] a esté rendue ". Il est intéressant de noter au passage que c'est le cas nantais qui a motivé les deux ordonnances de 1696 et 1725. D'ailleurs, dans les lettres patentes de Louis XVI confirmant les privilèges du Serment de France, à l'article III qui renseigne sur le statut des monnayeurs vis-à-vis du système des classes, il est indiqué que ceux-ci " sont exempts de l'ordre et discipline des classes et du service de nos Vaisseaux, en se conformant à l'Ordonnance du 5 juin $1725^{118}$ ". " Jurisprudence " nantaise ou profil de " matelot " monnayeur exclusivement nantais? L'étude de Bruno Collin sur l'atelier de Montpellier ferait plutôt pencher vers la première hypothèse ${ }^{119}$.

Des monnayeurs classés et donc a priori levés, s'ils naviguent : voilà pour la théorie. Voyons maintenant ce qu'il en est dans la pratique, en procédant à un recoupement entre les registres-matricules des classes et les réceptions à l'Hôtel des Monnaies, afin de confronter les années de levées avec l'année d'accession au Serment du monnayeur. Durant le premier tiers

116. Arch. dép. de Loire-Atlantique, B 5216, Lettre du 6 mars 1701, enregistrée au greffe le 15 septembre $1701, \mathrm{f}^{\circ} 27 \mathrm{r}^{\circ}-\mathrm{v}^{\circ}$.

117. Arch. dép. de Loire-Atlantique, B 5279, Ordonnance du Roy pour faire opter quelques particuliers de Nantes, du mêtier de la Monnoye, avec celuy de Matelot, 5 juin 1725.

118. Arch. mun. de Nantes, HH 24, pièce 8, Lettres patentes du Roi en forme d'édit portant renouvellement et confirmation des privilèges des Monnoyeurs, ajusteurs et tailleresses $d u$ Serment de France et des officiers des Monnoies, données à la Muette au mois d'octobre 1782 , p. 5 .

119. Bruno Collin cite en effet une ordonnance du 28 septembre 1741 rédigée sur le modèle des ordonnances nantaises de 1696 et 1725, mais destinée elle aux « ouvriers de la monnoie de Montpellier "... Coluın, Bruno, op. cit., p. 40-41. Des pêcheurs-monnayeurs languedociens? 
du siècle, une fois reçu monnayeur, le pêcheur n'est en principe plus levé. Plus levé, mais toujours classé. Cette attitude « bienveillante " pourrait s'expliquer par une lettre du secrétaire d'État à la Marine demandant à l'intendant de ne pas "inquiéter" les monnayeurs ${ }^{120}$.

Les campagnes que les monnayeurs ont effectuées, l'ont donc été avant leur réception à l'atelier monétaire. Mais compte tenu de l'âge moyen d'accession au Serment qui, comme on l'a vu, tourne autour de 20 ans ils sont en fait très peu nombreux à avoir fait des campagnes. Entre 1700 et 1732, période dominée par la guerre de Succession d'Espagne, il ne se trouve que quatre pêcheurs de Rezé, futurs monnayeurs, ayant participé à des campagnes sur les quatorze inscrits clairement identifiés. Deux ont fait une seule campagne, un pêcheur en a fait deux et un autre trois, mais ce dernier a 41 ans à l'ouverture du registre et a accédé au Serment très tardivement : il aurait été reçu monnayeur en 1695 à 36 ans ${ }^{121}$.

Les choses paraissent changer dès la fin du premier tiers du siècle. Rien alors ne semble plus distinguer les monnayeurs des autres pêcheurs. Face aux besoins de plus en plus importants de matelots pour ses vaisseaux dans le cadre de son conflit avec l'Angleterre, il n'est plus question à la Monarchie de faire preuve d'indulgence. Le cas d'André Ertaud est significatif de cette évolution. Reçu ajusteur en 1734, il participe quelques mois plus tard à une campagne sur L'Ardent et quelques années plus tard sert même 13 mois et 20 jours sur un navire de la compagnie des Indes ${ }^{122}$. Cette même année 1734, Guillaume Aubin, reçu ajusteur en 1727, effectue une campagne sur le Lys $^{123}$. Les monnayeurs se retrouvent donc à participer, comme les autres pêcheurs, aux campagnes de la guerre de Succession d'Autriche. Outre l'exemple d'André Ertaud, cité plus haut, on mentionnera le cas d'Yves Peillac. Reçu monnayeur en 1738, il est levé pour Brest en 1746. Il sera de retour en 1748 après un séjour dans les prisons d'Angleterre " ayant esté pris sur la frégate du Roy L'Embuscade ${ }^{124}$ ". Au moins Yves Peillac s'en est sorti vivant, ce qui n'est pas le cas de Guillaume Peillac, fils unique de Charles Peillac. Reçu monnayeur en 1744 et levé lui aussi en 1746, il meurt

120. Arch. mun. d'Indre, CC 3, Requête des monnayeurs " au Roy et à Nosseigneurs de Son conseil ", 27 avril 1762.

121. Arch. dép. de Loire-Atlantique, 120 J 192, Registres des matricules du quartier de Nantes, Matricules des matelots par classes, hors Nantes (1700-1732), f ${ }^{\circ} 43$; Arch. dép. de Loire-Atlantique, B 5295, Réception de Nicolas Dejoie, maître monnayeur, 12 avril 1698.

122. Arch. dép. de Loire-Atlantique, $120 \mathrm{~J}$ 201, Registre des matricules du quartier de Nantes, hors Nantes, Officiers mariniers et matelots (1739-1750), $\mathrm{f}^{\circ} 295$, matricule 62 ; Arch. dép. de Loire-Atlantique, B 5333, Réception d'André Ertaud, ajusteur, 15 février 1734.

123. Arch. dép. de Loire-Atlantique, $120 \mathrm{~J}$ 201, Registre des matricules du quartier de Nantes, hors Nantes, Officiers mariniers et matelots (1739-1750), $\mathrm{f}^{\circ} 285$, matricule 2; Arch. dép. de Loire-Atlantique, B 5327, Réception de Guillaume Aubin, maître ajusteur, 9 septembre 1728.

124. Arch. dép. de Loire-Atlantique, $120 \mathrm{~J}$ 201, Registre des matricules du quartier de Nantes, hors Nantes, Officiers mariniers et matelots (1739-1750), $\mathrm{f}^{\circ} 309$, matricule 145 ; Arch. dép. de Loire-Atlantique, B 5338, Réception d'Yves Peillac, ajusteur, 5 août 1738. 
peu après sur un vaisseau du Roi ${ }^{125}$. Durant la guerre de Sept Ans, conflit très gourmand en marins, les monnayeurs de Rezé apparaissent également logés à la même enseigne que les autres bargers : ainsi Alexandre-Julien Dejoie, reçu monnayeur en 1756, participe à deux campagnes, l'une en 1760, l'autre en $1762^{126}$. Les contestations des monnayeurs continuent mais les autorités ne cèdent pas. C'est en référence à l'ordonnance du 28 juillet 1746 portant " qu'aucun officier marinier et matelot ne pourra être exempté du service des vaisseaux de sa majesté sous prétexte de fonctions particulières " que le commissaire principal de la Marine de Nantes refuse d'accorder, durant la guerre d'indépendance américaine, l'exemption de service à Yves Chauvelon, demandée par une requête du prévôt des monnayeurs ${ }^{127}$.

Enfin, il apparaît qu'avant la réception du pêcheur au Serment, le système des classes peut jouer un rôle négatif en occasionnant un retard à la réception pouvant devenir a priori assez fâcheux pour le futur monnayeur. Dans le cadre du sondage effectué dans les réceptions à l'atelier, deux cas ont été relevés, deux victimes, si on peut dire, de la guerre de Sept Ans, tous deux postérieurs donc aux arrêts de 1755-1756, arrêts dont le délai limite de réception qu'ils imposent, oblige, en principe, tous postulants au Serment ou à la maîtrise à justifier d'un éventuel retard. Ce genre de mésaventure est arrivé ainsi à Nicolas Oresve en 1759. Âgé de 33 ans en 1759 - rappelons que la limite pour se faire recevoir est fixée à l'année qui suit le jour des 25 ans du postulant - il est pour le moins à un âge devenu théoriquement problématique pour être reçu au Serment, en l'occurrence ouvrier ajusteur. Il se justifie en indiquant " [qu']ayant esté plusieurs années au service du Roy dans la marine dont il n'est revenu que depuis très peu de temps, ayant esté prisonnier de guerre en Angleterre pendant plusieurs années et depuis son retour ayant esté malade des fatigues qu'il a eu, il n'a pas pu pendant ce temps perdre le droit qu'il a par sa naissance à la place d'ajusteur ". Justification lui permettant d'être reçu sans difficulté ${ }^{128}$. Même situation quelques jours plus tard pour Michel Aubin, âgé de 32 ans. Pour lui, la faute en revient aux 4 campagnes qu'il a enchaînées, mais cela ne l'empêchera pas de se faire finalement lui aussi recevoir ${ }^{129}$.

125. Arch. dép. de Loire-Atlantique, 120 J 201, Registre des matricules du quartier de Nantes, hors Nantes, Officiers mariniers et matelots (1739-1750), f ${ }^{\circ} 372$, matricule 193 ; Arch. dép. de Loire-Atlantique, B 5343, Réception de Guillaume Peillac, monnayeur, 11 août 1744 .

126. Arch. dép. de Loire-Atlantique, 120 J 210, Registre des matricules du quartier de Nantes, hors Nantes, Officiers mariniers et matelots (1751-1762), $\mathrm{f}^{\circ}$ 424, matricule 154 ; Arch. dép. de Loire-Atlantique, B 5353, Réception d'Alexandre-Julien Dejoie, maître monnayeur, 3 janvier 1760 .

127. Arch. dép. de Loire-Atlantique, C 963, Lettre du commissaire principal de la Marine à Nantes au ministre de la Marine, 12 octobre 1780 .

128. Arch. dép. de Loire-Atlantique, B 5352, Requête de Nicolas Oresve, 28 décembre 1759; accord de la chambre de l'ajusterie, 31 décembre 1759.

129. Arch. dép. de Loire-Atlantique, B 5353, Requête de Michel Aubin, 2 janvier 1760; Réception de Michel Aubin, ouvrier ajusteur, 3 janvier 1760. 
Notons enfin que les monnayeurs qui sont reçus pilotes se trouvent bien sûr exemptés du service des classes. Une " planque ", le pilotage? Concernant les bargers, c'est un soupçon assez récurrent chez les autorités ${ }^{130}$. Retenons donc que par ses deux ordonnances de 1696 et 1725 , le Roi a jugé que ses matelots monnayeurs lui étaient plus utiles sur ses vaisseaux qu'en sa Monnaie, avec cependant, dans la pratique, ce qui semble bien être un certain traitement de faveur dans le premier tiers du $\mathrm{XVIII}^{\mathrm{e}}$ siècle.

\section{Des monnayeurs chez les pêcheurs}

Population bénéficiant de privilèges fiscaux importants, les pêcheursmonnayeurs forment une notabilité non seulement au sein des pêcheurs de l'estuaire mais aussi au niveau paroissial. Une notabilité fière de sa différence, jalousée et attaquée par une partie de la population de leur paroisse, vivant en partie repliée sur elle-même et constituant ainsi une société dans la société des pêcheurs de l'estuaire.

\section{Des « honorables hommes »}

Les rôles de la capitation roturière sont un premier indicateur de la place de cette population au sein de la société des pêcheurs de l'estuaire, et de la société paroissiale dans son ensemble. À un demi-siècle d'intervalle, deux rôles de capitation de Rezé, l'un de $1710^{131}$, l'autre de $1762^{132}$, montrent le poids fiscal des monnayeurs.

Tableau 2 - Les monnayeurs du canton des Isles dans le rôle de la capitation roturière de Rezé pour les années 1710 et 1762

\begin{tabular}{|l|c|c|}
\hline & 1710 & 1762 \\
\hline Nombre de capités du canton des Isles 133 & 175 & 291 \\
Nombre de monnayeurs capités & 17 & 33 \\
Pourcentage des monnayeurs parmi les capités & $9,7 \%$ & $11,3 \%$ \\
Pourcentage des monnayeurs parmi les capités à 7 livres et plus & $50 \%$ & $49 \%$ \\
Pourcentage des monnayeurs capités à 7 livres et plus & $52,9 \%$ & $63,6 \%$ \\
\hline
\end{tabular}

130. À l'exemple du Général du commerce de Nantes : « les bargers de la Basse Indre dissimulent le véritable objet de leur demande d'être admis au pilotage : c'est pour s'exempter du service des classes de la marine ", Arch. dép. de Loire-Atlantique, L 1109, Avis des juges et consuls de Nantes, 6 décembre 1790 .

131. Arch. dép. de Loire-Atlantique, B 3499, Rôle de capitation de la paroisse de Rezé pour l'année 1710.

132. Arch. mun. de Rezé, CC 8, Rôle de capitation de la paroisse de Rezé pour l'année 1762.

133. Le canton fiscal des Isles est constitué, comme son nom l'indique, des îles de Rezé, c'est-à-dire de l'île des Chevaliers (avec les villages de Haute-Île et Basse-Île), le hameau de Nortiouze et l'île de Trentemoult, le tout représentant environ le quart des contribuables rezéens au début du XVIII siècle, et environ le tiers à la fin. 
En 1710, ne formant que 9,7 \% de la population capitée des îles, les monnayeurs représentent la moitié des capités à plus de 7 livres. Même proportion cinquante-deux ans plus tard : formant $11,3 \%$ de la population capitée des îles, ils représentent toujours la moitié des capités à 7 livres et plus. Sur ce rôle de 1762, les monnayeurs ne sont que 36,4 \% en dessous de la barre des 7 livres et donc 63,6 \% au-dessus. En prenant en comparaison l'ensemble des capités des îles, ces derniers sont 85,2\% en dessous de la barre des 7 livres et donc 14,7 \% au-dessus. Sur un rôle d'imposition intermédiaire, celui de la capitation de 1740, la cote moyenne des capités du canton est d'un peu plus de 4 livres, celle des pêcheurs-monnayeurs, pas loin du double, à un peu plus de 7 livres.

En rapportant maintenant la population des pêcheurs-monnayeurs à l'ensemble des capités de Rezé pour l'année 1762, voici les 10 premiers contribuables de la paroisse, sur 1022 capités, montrant là aussi une domination fiscale pour la moins nette des pêcheurs-monnayeurs.

\section{Tableau 3 - Les dix contribuables les plus imposés dans le rôle de la capitation roturière de Rezé pour l'année 1762}

\begin{tabular}{|l|c|l|l|l|}
\hline Nom & Âge & \multicolumn{1}{|c|}{ Profession } & \multicolumn{1}{|c|}{ Domiciliation } & \multicolumn{1}{c|}{ Imposition } \\
\hline André Dejoie & 50 ans & pêcheur-monnayeur & Trentemoult & 21 livres \\
\hline Étienne Lancelot & 50 ans & pêcheur & Trentemoult & 20 livres \\
\hline $\begin{array}{l}\text { César Silvestre } \\
\text { Chauvelon }\end{array}$ & 62 ans & pêcheur-monnayeur & Trentemoult & 19 livres 10 sols \\
\hline Simon Peillac & 79 ans & pêcheur-monnayeur & Haute-Île & 19 livres 10 sols \\
\hline Yves Peillac & 67 ans & $\begin{array}{l}\text { pêcheur, officier du } \\
\text { Serment de France }\end{array}$ & Haute-Île & 16 livres 10 sols \\
\hline René Peillac & 63 ans & pêcheur-monnayeur & Trentemoult & 15 livres 10 sols \\
\hline François Poidras & $?$ & marchand & Pont-Rousseau & 15 livres \\
\hline $\begin{array}{l}\text { La veuve Joseph } \\
\text { Chauvelon, pêcheur }\end{array}$ & $?$ & - & Trentemoult & 14 livres 10 sols \\
\hline Jean Giraud & $?$ & marchand & Port au Blé & 14 livres 10 sols \\
\hline François Chauvelon & 62 ans & pêcheur-monnayeur & Haute-Île & 14 livres 5 sols \\
\hline
\end{tabular}

Si on compare maintenant les cotes des pêcheurs-monnayeurs avec les autres monnayeurs rezéens non pêcheurs mais laboureurs, peu nombreux dans les rôles, huit en 1740 et sept en 1762, la cote moyenne est un peu près identique à celle des pêcheurs-monnayeurs, 7 livres pour 1740, mais les laboureurs-monnayeurs, contrairement aux pêcheurs, n'atteignent pas les plus hautes impositions de la paroisse.

Comme nous l'avons vu, les pêcheurs-monnayeurs sont moins nombreux à la veille de la Révolution. Ceux clairement identifiés dans le rôle de la capitation de $1789^{134}$ sont presque tous au-dessus de la barre des

134. Arch. dép. de Loire-Atlantique, B 3531, Rôle de capitation de la paroisse de Rezé pour l'année 1789. 
10 livres, le maximum étant de 21 livres pour Yves Peillac ${ }^{135}$. Avec une cote moyenne autour des 3 livres pour le canton des îles, ils représentent toujours une élite fiscale.

Face à ces chiffres montrant une prépondérance fiscale des pêcheursmonnayeurs dans les impositions les plus élevées de la capitation roturière de Rezé, il convient de s'interroger sur le niveau de représentativité de ces impositions sur le niveau de vie de ces pêcheurs-monnayeurs. L'élite sociale est-elle à la hauteur de l'élite fiscale? La question mérite d'être posée dans la mesure où, comme nous l'avons vu, il s'agit d'une population jalousée et exemptée des charges publiques : elle ne participe donc pas à la répartition de la capitation. On peut donc se demander si la répartition de la capitation ne se fait pas à son désavantage : autrement dit, cette population est-elle surimposée?

Encore qu'il convienne de rester prudent, cela ne semble pas le cas si l'on compare deux inventaires après décès de 1763, l'un d'un pêcheur, René Cassard ${ }^{136}$, l'autre d'un pêcheur-monnayeur, Jean Dejoie ${ }^{137}$ avec la capitation de 1762. Dans le cas de René Cassard, ce dernier a acquitté une capitation de 2 livres en 1762 (la moyenne du canton des îles cette année là est à 3 livres), et laisse un inventaire à 287 livres et 10 sols l'année suivante. Pour Jean Dejoie, qui a alors 56 ans, il s'agit d'un inventaire partiel après remariage ${ }^{138}$ d'une fortune donc quasi " aboutie ", s'élevant à 931 livres et 18 sols. Jean Dejoie a payé une capitation de 8 livres l'année précédente, se situant dans la moyenne des monnayeurs. Sur les 931 livres 18 sols de cet inventaire partiel, la literie représente 268 livres soit $28,8 \%$ du total, le matériel de pêche 322 livres soit $34,6 \%$ du total : ces deux éléments représentent donc plus de $60 \%$ de la valeur des biens déclarés.

Regardons donc de plus près la composition de la fortune mobilière de ce pêcheur-monnayeur que l'on peut considérer avec réserve comme "moyenne ". La literie est composée de 3 lits : l'un est un bois de lit à " quenouille " à 112 livres, les deux autres sont prisés respectivement 90 et 66 livres : cette literie semble donc dessiner le portrait d'une certaine indépendance économique. À cette dernière répond l'indépendance professionnelle, telle que le révèle le matériel de pêche. Jean Dejoie possède en effet deux barges prisées 60 livres et 210 livres de filets témoignant d'une activité de pêche aussi bien fluviale (lampresses, sédors) que maritime (filets "à harang "). De cet exemple, on peut déduire par comparaison que c'est sans doute en plusieurs milliers de livres que doivent se monter les inventaires potentiels des pêcheurs-monnayeurs les plus capités.

135. Le maximum pour le canton des îles en 1789 étant Étienne Lancelot pêcheur non monnayeur avec 31 livres.

136. Arch. dép. de Loire-Atlantique, B 6899/2, Inventaire René Cassard, 27 avril 1763

137. Arch. dép. de Loire-Atlantique, B 6899/2, Inventaire Jean Dejoie, 13 mai 1763.

138. Ses vêtements, séparés de la communauté, n'ont pas été inventoriés et ceux de sa femme sont partis habiller ses filles. 
Pour étudier le patrimoine foncier et immobilier, nous allons choisir un notable de l'île des Chevaliers représentant l'élite des pêcheurs-monnayeurs ${ }^{139}$. Avec 23 livres et 10 sols, Charles Peillac représente en 1758 le plus gros capité roturier de Rezé après une "veuve Tatin " à 36 livres ${ }^{140}$. Il décède en 1761 à l'âge de 81 ans, et un partage de ses biens a lieu en $1762^{141}$. La succession est divisée en 5 lots revenant à 310 livres 18 sols de revenu annuel, le tout formant un capital de fonds de 6838 livres. Voici d'abord un état de la superficie totale de ses terres ${ }^{142}$.

\section{Tableau 4 - Le patrimoine foncier du pêcheur-monnayeur Charles Peillac à sa succession en 1762}

\author{
Vignes (39\%) \\ Prés (30 \%) \\ Terres labourables (28\%) \\ Friches (3\%) \\ Total \\ Total (en hectares)
}
80 boisselées 6 gaulles
62 boisselées 13 gaulles
57 boisselées 8 gaulles
6 boisselées 59 gaulles
206 boisselées 26 gaulles
Environ 7,35 hectares

L'ensemble est très fragmenté. La répartition en superficie est relativement harmonieuse entre les prés, terres cultivables et vignes ("blanches"). L'essentiel se situe sur la paroisse de Rezé. Charles Peillac possède quelques terres dans les paroisses limitrophes : surtout à Saint-Sébastien (16 boisselées 8 gaulles), Bouguenais et ponctuellement Vertou et BasseGoulaine. C'est la vigne qui rapporte le plus. D'ailleurs, en recollant les différents lots, la parcelle la plus importante paraît être un clos de vigne de 42 boisselées constituant la terre au revenu annuel le plus important de la succession avec 30 livres.

Le patrimoine immobilier de Charles Peillac confirme une assise notable sur la paroisse de Rezé.

Tableau 5 - Le patrimoine immobilier du pêcheur monnayeur Charles Peillac à sa succession en 1762

\begin{tabular}{|l|l|l|l|}
\hline \multicolumn{1}{|c|}{$\begin{array}{c}\text { Descriptif du } \\
\text { bien immobilier }\end{array}$} & \multicolumn{1}{|c|}{ Situation } & \multicolumn{1}{c|}{$\begin{array}{c}\text { Obstimation } \\
\text { successorale }\end{array}$} \\
\hline $\begin{array}{l}\text { 1 Maison : } \\
\text { chambre basse + petit } \\
\text { cellier en appendice + } \\
\text { escalier de pierre + }\end{array}$ & $\begin{array}{l}\text { Haute-Ile des } \\
\text { Chevaliers } \\
\text { Rezé }\end{array}$ & $\begin{array}{l}\text { Demeure de } \\
\text { Charles Peillac }\end{array}$ & 540 livres \\
\hline
\end{tabular}

139. Pour la filiation de Charles Peillac, on peut se reporter au tableau en annexe. 140. Arch. mun. de Rezé, CC 7, Rôle de capitation de Rezé pour l'année 1758.

141. Arch. dép. de Loire-Atlantique, 4 E 22/29, Briand, Partage des biens de fonds dépendants de la succession de défunts Charles Peillac et Marguerite Ertaud sa femme, 15 février 1762.

142. Les mesures de Rezé sont celles du Comté de Nantes : la boisselée équivaut à 60 gaulles. Une boisselée correspond à $356,1318 \mathrm{~m}^{2}$. 
Des bargers monnayeurs au XVIII ${ }^{\mathrm{e}}$ siècle

\begin{tabular}{|c|c|c|c|}
\hline $\begin{array}{l}\text { chambre haute avec } \\
\text { cheminée + un grenier } \\
\text { (trappe + échelle) + } \\
\text { jardin de } 35 \text { gaulles }\end{array}$ & & & \\
\hline $\begin{array}{l}1 \text { Maison : } \\
\text { chambre basse + } \\
\text { chambre haute avec } \\
\text { cheminée + escalier de } \\
\text { pierre commun + } \\
\text { un grenier (trappe + } \\
\text { échelle) + jardin de } \\
11 \text { gaulles }\end{array}$ & $\begin{array}{l}\text { Haute-lle des } \\
\text { Chevaliers } \\
\text { Rezé }\end{array}$ & $\begin{array}{l}\text { Demeure de } \\
\text { Louis Peillac }\end{array}$ & 240 livres \\
\hline $\begin{array}{l}1 \text { Maison : } \\
\text { chambre basse + } \\
\text { hambre haute avec } \\
\text { cheminée + deux } \\
\text { escaliers de pierre } \\
\text { communs + un grenier } \\
\text { (trappe + échelle) + } \\
\text { jardin de } 9 \text { gaulles }\end{array}$ & $\begin{array}{l}\text { Haute-Ile des } \\
\text { Chevaliers } \\
\text { Rezé }\end{array}$ & $\begin{array}{l}\text { Demeure de } \\
\text { Julien Ollive } \\
\text { Un escalier de pierre } \\
\text { est commun avec la } \\
\text { maison précédente et } \\
\text { un autre avec } \\
\text { la suivante }\end{array}$ & 260 livres \\
\hline $\begin{array}{l}1 \text { Maison : } \\
\text { chambre basse + } \\
\text { chambre haute avec } \\
\text { cheminée + un escalier } \\
\text { de pierre commun + } \\
\text { un escalier de pierre } \\
\text { privatif + un grenier } \\
\text { (trappe + échelle) + } \\
\text { jardin de } 50 \text { gaulles }\end{array}$ & $\begin{array}{l}\text { Haute-lle des } \\
\text { Chevaliers } \\
\text { Rezé }\end{array}$ & $\begin{array}{l}\text { Demeure de } \\
\text { Julien Chauvelon }\end{array}$ & 420 livres \\
\hline $\begin{array}{l}1 \text { Maison : } \\
\text { chambre basse avec } \\
\text { cheminée + chambre } \\
\text { au derrière servant } \\
\text { d'écurie + grenier } \\
\text { au-dessus des deux } \\
\text { chambres (échelle + } \\
\text { trappe) + toit à cochon } \\
\text { derrière dernière } \\
\text { chambre + jardin de } \\
32 \text { gaulles } \\
\end{array}$ & $\begin{array}{l}\text { Port au Blé } \\
\text { Rezé }\end{array}$ & - & Rezé \\
\hline $\begin{array}{l}1 \text { Maison : } \\
\text { chambre basse avec } \\
\text { cheminée et degré à } \\
\text { noyau qui sert pour } \\
\text { monter dans un grenier } \\
\text { qui est au-dessus + } \\
\text { petite cour + appendice } \\
\text { avec pressoir et petit } \\
\text { cellier + jardin d'une } \\
\text { boisselée } 42 \text { gaulles }\end{array}$ & $\begin{array}{l}\text { Village de } \\
\text { la Petite Lande } \\
\text { Rezé }\end{array}$ & - & 300 livres \\
\hline
\end{tabular}

On notera la morphologie particulière des habitations des îles rezéennes, que cela soit celles des Chevaliers ou de Trentemoult. C'est un habitat construit contre les eaux de la Loire : les maisons, dont les couvertures sont en tuiles, sont généralement sur trois niveaux. Le premier est 
occupé par le cellier, la pièce d'habitation étant au deuxième niveau : comme on le voit c'est effectivement au deuxième que l'on trouve les cheminées. Le dernier niveau est occupé par un grenier qui peut parfois communiquer avec les greniers mitoyens. Les escaliers de pierre sont en principe extérieurs, parfois doublés par un escalier intérieur.

Ainsi, les pêcheurs-monnayeurs, pas tous mais un grand nombre ${ }^{143}$, forment $\mathrm{au} \mathrm{XVIII}{ }^{\mathrm{e}}$ siècle une véritable " aristocratie fluviale " dominant la notabilité, roturière, de Rezé. Le choix a été fait ici de se concentrer sur Rezé, qui regroupe l'essentiel des pêcheurs-monnayeurs et non Indre : il faudrait comparer le niveau de vie des monnayeurs d'Indre avec celui des monnayeurs de Rezé. Corinne Lodé remarque " qu'ils vivent aisément ${ }^{144}$ " et le général de paroisse d'Indre considère, sans doute avec quelque exagération, "que les monnoyeurs sont les plus riches habitants de la paroisse ${ }^{145}$ ". Notables nés des privilèges, ces derniers cultivent leur différence et leur position dominante: les pêcheurs-monnayeurs constituent un milieu fermé dans la société déjà très peu ouverte du monde des pêcheurs de l'estuaire de la Loire.

\section{Une société cloisonnée}

Le mode d'admission au sein du Serment, la situation insulaire de ces monnayeurs au sein d'une société de pêcheurs caractérisée traditionnellement par une forte reproduction sociale, ne peuvent entretenir a priori qu'une petite communauté cloisonnée, repliée sur elle-même. L'approche proposée ici se base sur la famille rezéenne des Peillac, dont 24 monnayeurs ont été identifiés pour le XVIII ${ }^{\mathrm{e}}$ siècle, et essentiellement sur la descendance sur deux générations de Sébastien Peillac (1654-1725) ${ }^{146}$.

C'est effectivement un monde assez clos qui se dessine. Sur les 15 mariages de cette descendance, au moins 7 ont été contractés avec des monnayeurs, effectifs ou en droit, ou des filles de monnayeurs. Tous les mariages pour lesquels la qualité professionnelle de l'époux, ou celle du père pour les mariées, a pu être identifiée, ont été contractés entre pêcheurs. Des pêcheurs-monnayeurs notables se tournant vers des pêcheurs non monnayeurs mais notables aussi : dans le cadre de la descendance de Sébastien Peillac, on relève le mariage entre son fils Antoine et Elizabeth Lancelot, appartenant à une famille de gros maîtres de barge de Trentemoult ${ }^{147}$. Il convient cependant de faire une distinction entre les

143. Il ne faudrait pas oublier ces quelques pêcheurs-monnayeurs dont les privilèges n'ont peut-être pour seul apport que d'empêcher ces derniers de tomber dans la misère. 144. LoDE, Corinne, op. cit., p. 38.

145. Arch. mun. d'Indre, CC 3, Requête des monnayeurs " au Roy et à Nosseigneurs de Son conseil ", 27 avril 1762.

146. Voir tableau en annexe. Arch. dép. de Loire-Atlantique, 1 Mi EC 386, Registres paroissiaux de Rezé. Nous remercions Jean-Claude Cahagne pour la communication de ses recherches généalogiques.

147. Arch. dép. de Loire-Atlantique, 1 Mi EC 386, Registres paroissiaux de Rezé, Mariage de Catherine Lancelot et René Peillac, 3 juillet 1719; mariage d'Élisabeth Lancelot et Antoine Peillac, 27 août 1708 . 
deux sexes. Les deux fils de Sébastien Peillac se sont mariés tous deux avec une fille de monnayeurs, par contre les deux filles se sont mariées avec des pêcheurs extérieurs au Serment. L'explication en est simple : les filles de monnayeurs sont toutes, comme on l'a vu, des tailleresses potentielles, elles peuvent donc communiquer, par leurs futurs enfants, le droit d'entrée à la Monnaie et surtout ce qui va avec, les privilèges, à une famille qui en était jusque là exclue. On imagine aisément le père d'une tailleresse potentielle profiter des vertus monétaires de sa fille pour négocier une dot qui lui est avantageuse avec une famille étrangère au Serment. Peut-être qu'une étude comparative de contrats de mariage pourrait illustrer ce comportement, étude assez délicate à mener car il faudrait comparer des niveaux de vie similaires. À côté d'une ouverture contrainte et relative, se limitant aux autres pêcheurs, et due au faible nombre de monnayeurs, les tailleresses constituent donc un autre élément d'ouverture, relatif aussi, des monnayeurs sur le reste de la société des pêcheurs.

Non seulement on se marie entre pêcheurs-monnayeurs, et entre pêcheurs faute de monnayeurs, mais on reste entre insulaires : dans l'ensemble des mariages identifiés concernant les Peillac rezéens au XVIII siècle et avant la Révolution, c'est-à-dire 42 mariages, les époux sont originaires tous deux des " isles " de Rezé dans la quasi-totalité des cas, c'est-à-dire dans au moins 37 mariages. On relève un mariage en 1767 avec un non rezéen : il s'agit de l'union entre Françoise Peillac et Jacques Saulny, barger d'Indre. Une exception car les relations matrimoniales entre les deux communautés estuariennes de pêcheurs-monnayeurs semblent assez rares ${ }^{148}$.

Révélatrices d'une communauté étroite et repliée : les dispenses de consanguinité. Dans la descendance Sébastien Peillac, 3 mariages sur les 15 en ont nécessité une au $3^{\mathrm{e}}$ ou $4^{\mathrm{e}}$ degré. Elles concernent toutes la branche de son fils cadet, Charles Peillac.

Pour cette société de monnayeurs dans une société de pêcheurs insulaires, que cela soit à Indre ou Rezé, la reproduction sociale est donc très forte, cependant, comme nous avons déjà eu l'occasion de le souligner, l'absence des professions dans les registres paroissiaux et les rôles fiscaux de Rezé rend bien difficile l'identification des nuances à apporter. Heureusement, différentes autres sources, comme les actes notariés ou même les registres-matricules, peuvent fournir ponctuellement ces nuances. Dans cette chaîne reproductrice de pêcheurs, quelques cas particuliers peuvent en effet être relevés. Trois cas établissent des jonctions avec le monde des boulangers, ce dernier pouvant parfois constituer une petite notabilité artisanale : c'est avec Jean Aguesse, maître boulanger de Rezé que Jeanne Peillac, fille de Simon Peillac se marie en $1732^{149}$, le fils unique de César Silvestre Chauvelon, maître monnayeur, est maître boulanger à L'Hermitage

148. Arch. dép. de Loire-Atlantique, 1 Mi EC 386, Registres paroissiaux de Rezé, Mariage de Jacques Saulny et Françoise Peillac, 25 août 1767.

149. Arch. dép. de Loire-Atlantique, 1 Mi EC 386, Registres paroissiaux de Rezé, Mariage de Jean Aguaisse et Jeanne Peillac, 6 mai 1732. 
à Chantenay ${ }^{150}$ et Louis-Guillaume, fils aîné du prévôt des monnayeurs Guillaume Peillac, monnayeur lui aussi, est signalé en 1747 comme apprenti boulanger ${ }^{151}$. Un cadet de trop? C'est vers le métier de poêlier que se destine Augustin Ollive, ajusteur, fils cadet de pêcheur et de tailleresse, frère de pêcheur-monnayeur. On le retrouve par la suite installé à Nantes ${ }^{152}$ avant de partir à Saint-Domingue comme engagé, à 39 ans ${ }^{153}$.

Le parrainage des enfants de pêcheurs-monnayeurs confirme également ce cloisonnement social. Les parrains et marraines appartiennent aux familles de pêcheurs-monnayeurs rezéens, parfois de pêcheurs-monnayeurs non rezéens : on retrouve ainsi chez les Peillac les liens qui unissent cette famille rezéenne avec les Saulny d'Indre. Lors du baptême d'Anne-Jeanne Peillac, fille de Simon-Pierre Peillac et d'Anne Ménager, en 1774, c'est Jacques Saulny, cité plus haut, oncle par alliance de l'enfant au maternel, qui est le parrain ${ }^{154}$. Quand le parrain ou la marraine n'appartiennent pas à des familles de pêcheurs-monnayeurs, on les trouve chez des notables, parfois également monnayeurs : en 1714, lors du baptême de Marie Peillac, fille de Simon Peillac, c'est Christophe Phelippes, notaire royal et monnayeur qui est le parrain et la fille de ce dernier la marraine ${ }^{155}$. Du côté des pêcheurs non-monnayeurs, on retrouve la famille Lancelot : lors du baptême d'Anne Peillac en 1728, c'est Étienne Lancelot qui est le parrain ${ }^{156}$. Pêcheurs-monnayeurs notables, ces derniers trouvent des parrainages à la fois dans la haute administration locale de la Marine et dans celle de la Monnaie. Lors du baptême d'Élisabeth Peillac, fille d'Antoine, le 22 novembre 1706, le parrain n'est autre que le fils du directeur de la Monnaie de Nantes ${ }^{157}$ et lors du baptême en 1731 de Marie-Françoise Peillac, fille de Silvestre Peillac, c'est un commissaire de la Marine aux classes du département de Nantes qui est le parrain et la femme du commissaire principal des classes du département de Nantes qui en est la marraine ${ }^{158}$.

150. Arch. dép. de Loire-Atlantique, 4 E 22/32, Briand, Transaction entre Françoise Clouet veuve de César Silvestre Chauvelon et les enfants de son mari, 28 juin 1769.

151. Arch. dép. de Loire-Atlantique, 120 J 201, Registre des matricules du quartier de Nantes, Officiers mariniers et matelots, Autres paroisses (1739-1750), Rezé, f ${ }^{\circ}$ 312, matricule 165.

152. Arch. dép. de Loire-Atlantique, 4 E 2 280/2, Bertrand, Marché d'apprentissage de poêlier pour 300 livres entre Louis Garot, poêlier, et la veuve de Blaise Ollive, $1^{\mathrm{er}}$ août 1747; 4 E 22/28, Briand, Partage des biens de fonds dépendant des successions de défunts Blaise Ollive et Marie Peillac sa femme fait entre leurs enfants, 17 août 1760. Voir tableau en annexe.

153. Arch. dép. de Loire-Atlantique, 120 J 415, Rôle d'armement de l'Arcahaye, 1764.

154. Arch. dép. de Loire-Atlantique, 1 Mi EC 386, Registres paroissiaux de Rezé, Baptême du 10 avril 1774.

155. Arch. dép. de Loire-Atlantique, 1 Mi EC 386, Registres paroissiaux de Rezé, Baptême du 15 septembre 1714.

156. Arch. dép. de Loire-Atlantique, 1 Mi EC 386, Registres paroissiaux de Rezé, Baptême du 26 juillet 1728 .

157. Arch. dép. de Loire-Atlantique, 1 Mi EC 386, Registres paroissiaux de Rezé, Baptême du 22 mars 1706.

158. Arch. dép. de Loire-Atlantique, 1 Mi EC 386, Registres paroissiaux de Rezé, Baptême du 14 avril 1731. 
Une société de pêcheurs-monnayeurs donc fortement endogame. L'ouverture relative se limite aux autres pêcheurs des îles et a deux causes : le nombre restreint de monnayeurs et le " commerce " que le père monnayeur peut faire avec une fille ayant dans sa corbeille nuptiale les privilèges de la Monnaie. Il a été signalé plus haut les relations entre les monnayeurs, tout du moins les plus importants d'entre eux et les Lancelot, gros maîtres pêcheurs, non monnayeurs, de Trentemoult, personnages influents, dispensateurs du crédit local et surtout très régulièrement présents au sein du général de paroisse, alliance sans doute précieuse car c'est fort logiquement au niveau de ce dernier que prend forme la lutte à l'échelle paroissiale contre les monnayeurs.

\section{Haro sur les monnayeurs}

Il s'agit ici, pour finir, de proposer une première approche des conflits opposant les monnayeurs et les autres habitants des paroisses d'Indre et de Rezé par un effleurement des sources judiciaires, tant ces dernières paraissent prometteuses. C'est au niveau du général de paroisse, dont les monnayeurs sont exclus de par leurs privilèges, que la lutte contre ces derniers s'organise au niveau paroissial. Des monnayeurs malencontreusement inscrits sur les rôles des fouages ou nommés collecteurs forment des affaires judiciaires récurrentes à Rezé et à Indre : tel est le cas, par exemple, à Rezé, de François Bertrand en 1731, imposé de 6 sols " dans le rôle des soudes et fouages ${ }^{159}$ ". Silvestre Peillac, confronté au même problème, dépose une requête au Présidial et obtient gain de cause. Le général de Rezé " pour éviter la taxe des dépens du présidial " fait alors appel au Parlement ${ }^{160}$. Procédure pouvant être fort longue, puisqu'il est fait mention d'un autre procès contre les monnayeurs " actuellement indécis au parlement de cette province ", vieux semble t-il d'un certain nombre d'années ${ }^{161}$. Pour Indre, on peut citer, par exemple, le cas de Louis-Luc Oresve et Clair Saulny qui obtiennent une sentence de décharge des commissaires des États de Bretagne en 1761, parce que le premier s'est trouvé nommé égailleur et le second collecteur du vingtième ${ }^{162}$. Un affrontement qui n'est nullement propre au XVIII ${ }^{\mathrm{e}}$ siècle : les sources judiciaires de ce siècle gardent en effet la mémoire de luttes lointaines ${ }^{163}$.

\footnotetext{
159. Arch. mun. de Rezé, BB 1, Délibérations du général de paroisse, 13 janvier 1732, $\mathrm{f}^{\circ} 43 \mathrm{v}^{\circ}-\mathrm{f}^{\circ} 44 \mathrm{r}^{\circ}$.

160. Arch. mun. de Rezé, BB 1, Délibérations du général de paroisse, 6 avril $1732, \mathrm{f}^{\circ} 46 \mathrm{v}^{\circ}$.

161. Arch. mun. de Rezé, BB 1, Délibérations du général de paroisse, 24 février 1732, $\mathrm{f}^{\circ} 45 \mathrm{v}^{\circ}$.

162. Arch. mun. d'Indre, CC 3, Sentence de décharge des commissaires des États de Bretagne, 19 mai 1761.

163. Ainsi cet arrêt du Parlement de Bretagne du 27 octobre 1563 en faveur des monnayeurs de Nantes et de Rennes contre les habitants de la paroisse de Pacé " qui vouloient les assujettir aux fouages ", Arch. mun. d'Indre, CC 3, Requête des monnayeurs "au Roy et à Nosseigneurs de Son conseil ", février 1759.
} 
Les privilèges fiscaux ont en effet de quoi irriter les autres paroissiens : les monnayeurs sont autant de contribuables en moins qui font peser de fait plus lourdement une répartition des fouages sur les autres contribuables. La traduction judiciaire de l'opposition entre les généraux de paroisse et les monnayeurs peut se résumer en une question jouant sur une ambiguïté juridique : les monnayeurs doivent-ils payer les fouages sur leurs acquêts? Pour les généraux de paroisse, soutenus par les États de Bretagne, la réponse est oui; pour les monnayeurs, on s'en doute, la réponse est non. Les généraux de paroisse ont un allié : les États de Bretagne, qui lèvent les fouages. En 1726, des lettres patentes portant règlement pour la levée des fouages en Bretagne sont publiées ${ }^{164}$. Le fait que quelques privilégiés, dont les monnayeurs, aient été manifestement oubliés dans ses lettres, ne peut qu'entraîner une suite de procédures judiciaires : c'est ainsi que les États considèrent dans une instance au Parlement, "que tous [les] biens [des monnayeurs] en général ne sont point exempts des fouages, il est constant qu'ils n'en n'ont l'exemption que par rapport à leurs biens de patrimoine; leurs biens d'acquêts sont sujets aux fouages; les livres sont pleins d'arrêts qui l'ont décidé même en 1555 en faveur du général de Saint-Sébastien ${ }^{165}$ ". Dans un mémoire ${ }^{166}$ rédigé contre trois corps d'exemptés " oubliés " par les lettre patentes de 1726, c'est-à-dire "les Suppôts de l'Université ", "les monnoyeurs " et " les habitans de la ville de Nantes ", les États, après avoir constaté, avec quelques exagérations, les monnayeurs " pullulent et se multiplient immodérément, [que] la Monnoye de Nantes travaille peu, et [que] cependant cette Ville, ses Fauxbourgs et Campagnes voisines, sont pleins de gens soi disant Monnoyeurs ", insistent, après avoir demander une réduction de leur effectif, sur l'existence d'arrêts " dont quelques-uns réduisent l'Exemption au patrimoine des Monnoyeurs, et ne l'extendent pas à leurs acquêts ". Ils sont entendus par les avocats au Parlement, qui, s'ils ne les suivent pas sur la réduction des effectifs, constatent " qu'il est certain qu'ils n'ont d'exemption que par rapport à leurs biens patrimoniaux et ceux de leurs femmes, et qu'ils payent leurs fouages sur leurs acquêts, ou du moins ils doivent les payer ${ }^{167}$ ".

On ne sait si les États ont été un " relais " efficace pour les généraux de paroisse, mais c'est principalement sur ce point des acquêts que le général de paroisse d'Indre, dont l'exemple va être pris ici, décide en 1749 d'imposer sur les rôles des fouages l'ensemble des monnayeurs d'Indre, c'est-

164. Arch. dép. de Loire-Atlantique, C 15, Lettre patentes du Roy sur arrest du Conseil portant Règlement pour la levée des foüages en Bretagne, données à Versailles le 20 août 1726.

165. Arch. dép. d'Ille-et-Vilaine, C 2717, Greffe des États de Bretagne (1736-1738), Instance au Parlement sur les requêtes et lettres de l'omission prises par le général de Saint-Sébastien contre les Etats deffendeurs dans un procès que le général a en la cour contre plusieurs habitants de la ville de Nantes qui se prétendent exempts des foüages, non datée, $\mathrm{f}^{\circ}$ 166-167. 166. Arch. dép. d'Ille-et-Vilaine, C 2717, Greffe des États de Bretagne (1736-1738), Mémoire des Estats de Bretagne sur lequel il demande l'avis du conseil, non daté, $\mathrm{f}^{\circ}$ 179-182.

167. Arch. dép. d'Ille-et-Vilaine, C 2717, Greffe des États de Bretagne (1736-1738), Consultation des avocats au Parlement de Bretagne sur le mémoire des Estats concernant l'exemption des foüages prétendües par différentes personnes, 27 avril 1737, $\mathrm{f}^{\circ} 183$. 
à-dire une dizaine de personnes, presque tous bargers, formant une communauté de monnayeurs, par sa faiblesse numérique, plus vulnérable que celle de Rezé. Pour se justifier, le général dit que ce sont des acquêts que les monnayeurs " ont acquis en différens temps à la faveur de leur industrie dans des professions étrangères à la monoye [...] qui les mettent en état de faire tous les jours des acquêts dans la paroisse d'Indre et dans les voisines ${ }^{168}$ ". Les monnayeurs déposent alors une requête au Présidial mais ne réussissent pas à obtenir un jugement « que les habitants d'Indre pressentant qu'il ne leur seroit pas favorable ont empêché qu'il fut rendu ${ }^{169}$ ". Les monnayeurs s'adressent donc là où ils sont sûrs d'obtenir gain de cause : la cour des Monnaies de Paris. Un arrêt est rendu en 1752, sans surprise, en leur faveur, ignoré par le général de paroisse qui a " exercé contre eux les poursuites les plus violentes pour les forcer à payer ${ }^{170}$ ". Le général de paroisse est alors assigné devant la Cour des Monnaies et un nouvel arrêt est rendu le 2 juillet 1755 ordonnant le remboursement des sommes. Mais les habitants d'Indre " pour faire diversion et fatiguer les suppliants ${ }^{171}$ ", se pourvoient devant le conseil des Finances par une requête et obtiennent à leur tour un arrêt, mais du Conseil d'État celui-là, en leur faveur, le 9 septembre $1755^{172}$. À charge de revanche, puisque les monnayeurs obtiennent un nouvel arrêt du Conseil d'État, le 5 février 1760, confirmant leur privilège d'exemption des fouages ${ }^{173}$.

Derrière ces luttes judiciaires, pour ne pas dire parfois des chicaneries, souvent stériles, entretenues par les rivalités institutionnelles et les contradictions de l'administration royale, et qui finissent par être coûteuses de part et d'autre, se dessinent donc, d'une part, la détermination des monnayeurs à défendre leur singularité, ces privilèges fiscaux objets de fierté, qui les élèvent au-dessus des autres roturiers; d'autre part, la détermination des généraux de paroisse à lutter contre ce qui est vécu comme une profonde injustice.

Ajoutons enfin pour finir que si les monnayeurs constituent une petite communauté repliée, dans un environnement plutôt hostile, cela ne signifie pas bien sûr des solidarités sans failles : solidaires face à l'adversaire, ils ne le sont pas forcement entre eux! Ainsi, en 1728, près de Saint-Jeande-Boiseau, ce sont deux maîtres-monnayeurs de Trentemoult, Robert Boju et Silvestre Peillac, chacun dans leur barge avec leur compagnon, celui de Boju étant aussi monnayeur, qui se menacent, avec même une tentative

168. Arch. mun. d'Indre, CC 3, Défenses que fournit au siège Présidial de Nantes le général de la paroisse d'Indre, 13 février 1750.

169. Arch. mun. d'Indre, CC 3, Requête des monnayeurs " au Roy et à Nosseigneurs de Son conseil ", février 1759.

170. Idem.

171. Id

172. Arch. nat., $\mathrm{H}^{1}$ 606, Arrêt du Conseil d'État du 9 septembre 1755.

173. Arch. mun. d'Indre, CC 3, Requête des monnayeurs " au Roy et à Nosseigneurs de Son conseil ", 27 avril 1762; Arch. dép. de Loire-Atlantique, B 5283, Arrêt du Conseil d'État du 5 février 1760 . 
d'abordage de Silvestre Peillac " une gaffe à la main " qui aurait dit à son compagnon " tiens bon la barge que j'assomme ces bougres-là ${ }^{174}$ ".

Cette première approche aura donc tenté de dégager quelques caractéristiques fondamentales de cette population de pêcheurs-monnayeurs dont la première est la mise en évidence d'un profil de notable du fleuve, notable d'une " aristocratie roturière " enfermée, jalousée et attaquée. Il s'agit ici d'une pluriactivité bien particulière, puisque ce n'est pas l'activité du monnayage en tant que telle qui est recherchée, mais ce qui lui est attachée : des privilèges. Une biactivité donc toute relative, même si, comme on l'a vu, on aurait bien tort de mettre sur le même plan un laxisme des pêcheurs et l'absentéisme généralisé des faux ouvriers que sont les bourgeois en recherche de noblesse. Mais ces privilèges font-ils à eux seuls la notabilité? Certainement pas, dans la mesure où certains monnayeurs restent manifestement en dehors de ce processus de "notabilisation ". Une affaire de privilèges, certes, mais sans doute aussi une affaire d'hommes, de familles et de "réseaux".

De nombreuses questions restent bien sûr en suspens. Il faudrait étudier cette population pour les siècles antérieurs et par-là s'interroger sur ses origines, mais encore faut-il que les sources le permettent ${ }^{175}$. Les relations entre les activités fluvio-maritimes et celle du monnayage pourraient certainement être approfondies par une exploitation minutieuse du très riche fonds judiciaire et des fonds parisiens ${ }^{176}$. Il serait également intéressant d'étudier l'attitude des (ex-)monnayeurs à partir de la Révolution, notamment vis-à-vis des charges publiques dont ils étaient exemptés ${ }^{177}$, ainsi que le rôle de leurs descendants dans la mutation des activités fluviomaritimes non seulement rezéennes mais aussi nantaises et on touche ici un point essentiel.

174. Arch. dép. de Loire-Atlantique, B 4941, Plainte de Robert Boju et Julien Dejoie, 9 novembre 1728 .

175. À titre d'exemple, à partir des registres paroissiaux, des sources notariales et fiscales, des lettres d'interruption, des monnayeurs sont identifiables dans la seconde moitié du XvI ${ }^{\mathrm{e}}$ siècle dans trois familles de pêcheurs : les Peillac, Ertaud et Chauvelon, puis la nuit archivistique semble alors tomber sur eux. Pour la période antérieure, en effet, les sources deviennent particulièrement problématiques dès qu'il s'agit d'identifier un monnayeur qui n'appartient pas à la bourgeoisie, ce que souligne Yves Coativy : " nous ne savons pour ainsi dire rien des origines sociales des monnayeurs, en dehors des courtisans ", COATIVY, Yves, op. cit., p. 262-263.

176. On pense d'abord au fonds de la Cour des Monnaies, conservé aux Archives nationales, sous-série $\mathrm{Z}^{1 \mathrm{~b}}$.

177. Jean-Baptiste Ertaud, par exemple, ancien monnayeur d'une branche " terrienne ", est maire de Rezé de 1803 à 1807. La "radicalité " de la coupure révolutionnaire entre la Monnaie et le personnel de l'ancien Serment mériterait peut-être d'être nuancée. Ainsi, cet Ertaud signalé comme " contrôleur du monnoyage " à l'Hôtel des Monnaies en l'an XI. La suppression de la corporation et des privilèges ne signifie pas forcément disparition totale du monnayeur d'Ancien Régime. Arch. dép. de Loire-Atlantique, PER 51/1, Etrennes nantaises pour l'an XI (1802-1803), p. 64. 
Cette approche des pêcheurs-monnayeurs a débuté par la citation d'une affaire judiciaire mettant en scène un Chauvelon, Silvestre de son prénom. Elle va être refermée par l'évocation d'un autre Chauvelon, trentemousin lui aussi, mais né à la fin du siècle suivant.

Lorsque Julien Chauvelon prend le commandement du Belem en 1900, commandement qu'il conservera pendant 13 ans, sa situation professionnelle ne fait que s'inscrire alors dans ce qui est devenu une véritable tradition maritime rezéenne. C'est en effet tout un corps de capitaines " antillais " ou " cap-horniers ", sans compter un contingent particulièrement important de maîtres au cabotage, que Trentemoult fournit au port de Nantes depuis plusieurs décennies. Village alors pleinement maritime, il est déjà loin le temps où les îles de Rezé dominaient, écrasaient même, les activités de la pêche fluviale nantaise. Quelle mutation en un peu plus d'un siècle! Les " isles " de pêcheurs se sont muées en un foyer essentiel de recrutement d'officiers de commerce pour le port de Nantes. Et pourtant, les mêmes familles de notables du XVIII ${ }^{\mathrm{e}}$ siècle sont toujours présentes à la fin du XIX ${ }^{\mathrm{e}}$ siècle. Elles ont donc été capables, pour beaucoup d'entreelles, de s'adapter à l'évolution régionale du contexte économique fluviomaritime, d'en être même un des moteurs. L'évolution professionnelle des Chauvelon, Ertaud ou autres Dejoie en est l'illustration : celui du passage réussi d'une notabilité fluviale que sont les pêcheurs-monnayeurs au XVIII ${ }^{\mathrm{e}}$ siècle à une notabilité maritime que seront leurs descendants au $\mathrm{XIX}^{\mathrm{e}}$ siècle ou comment on est passé de la Monnaie au cap Horn.

\section{RÉSUMÉ}

Au XVIII ${ }^{\mathrm{e}}$ siècle, les monnayeurs nantais sont pour la plus forte minorité d'entre eux des pêcheurs. L'appartenance de ces pêcheurs à la corporation des monnayeurs, appelée le Serment de France et dont le recrutement se fait par filiation, procure à ces derniers d'importants privilèges fiscaux. Ce sont ces privilèges qui motivent les pêcheurs à se faire recevoir à l'atelier monétaire et non la recherche d'un travail complémentaire, aléatoire et très mal rémunéré.

Dans les paroisses d'Indre et surtout de Rezé, les pêcheurs-monnayeurs constituent une véritable " aristocratie ", dominant la notabilité roturière, et doivent affronter la jalousie du reste de la population.

\section{ABSTRACT}

In the $18^{\text {th }}$ Century, the fishermen constitute the strongest minority of the Nantes coiners. The membership of these fishermen to the corporation of the coiners, called the Oath of France and whose recruitment is done by filiation, their get significant fiscal privileges. These are the latter which motivate the fishermen to be made receive at the monetary workshop and not research of complementary work, random and very badly remunerated.

In the parishes of Indre and especially of Rezé, the fishermen-coiners constitute a true "aristocracy", dominating the commoner notability, and must face the jealousy of the remainder of the population. 


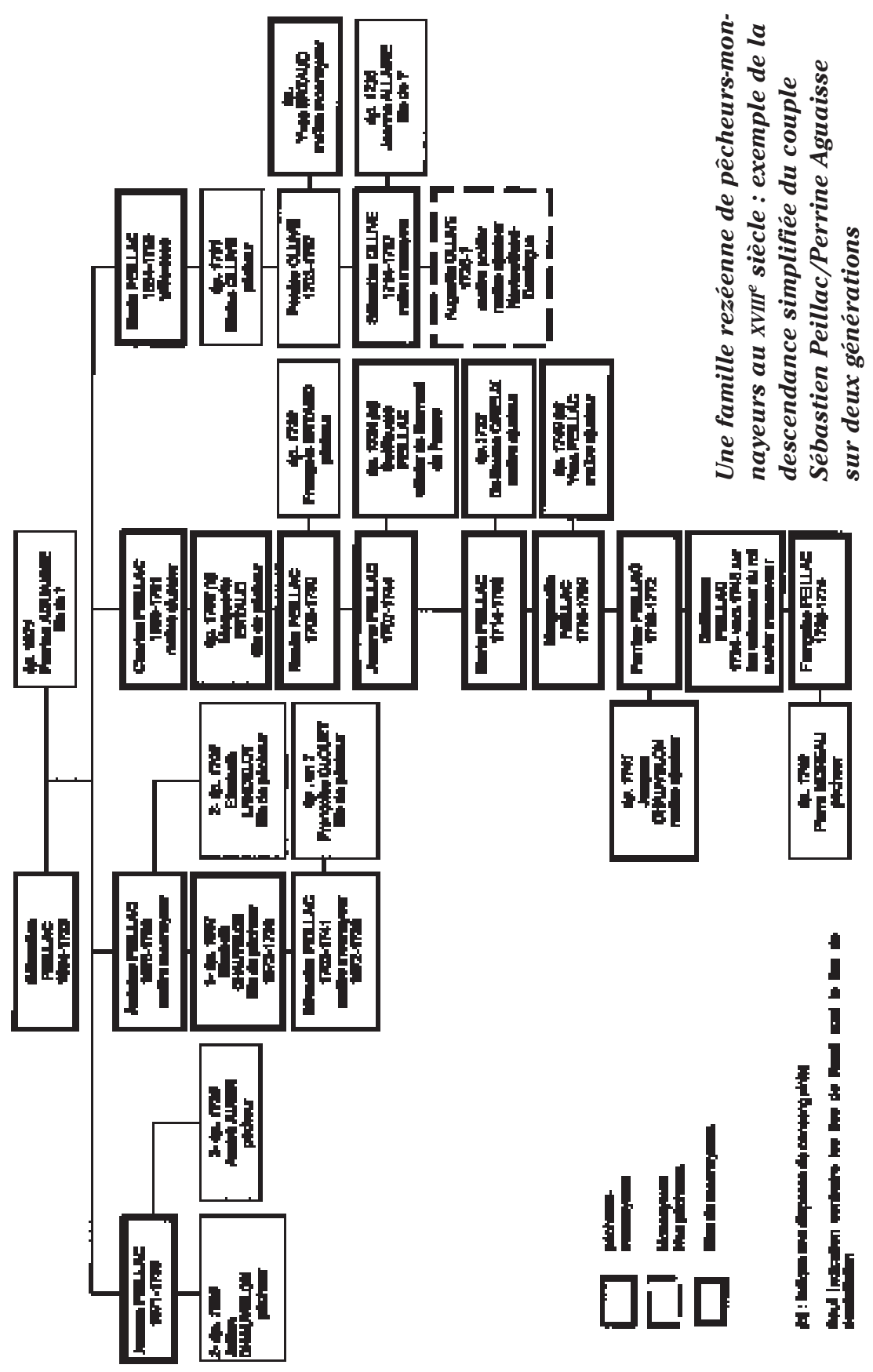

